\title{
Efficient Secure and Verifiable Outsourcing of Matrix Multiplications
}

\author{
Yihua Zhang and Marina Blanton \\ Department of Computer Science and Engineering \\ University of Notre Dame \\ yzhang16@nd.edu, mblanton@nd.edu
}

\begin{abstract}
With the emergence of cloud computing services, a resource-constrained client can outsource its computationally-heavy tasks to cloud providers. Because such service providers might not be fully trusted by the client, the need to verify integrity of the returned computation result arises. The ability to do so is called verifiable delegation or verifiable outsourcing. Furthermore, the data used in the computation may be sensitive and it is often desired to protect it from the cloud throughout the computation. In this work, we put forward solutions for verifiable outsourcing of matrix multiplications that favorably compare with the state of the art. Our goal is to minimize the cost of verifying the result without increasing overhead associated with other aspects of the scheme. In our scheme, the cost of verifying the result of computation uses only a single modulo exponentiation and the number of modulo multiplications linear in the size of the output matrix. This cost can be further reduced to avoid all cryptographic operations if the cloud is rational. A rational cloud is neither honest nor arbitrarily malicious, but rather economically motivated with the sole purpose of maximizing its monetary reward. We extend our core constructions with several desired features such as data protection, public verifiability, and computation chaining.
\end{abstract}

\section{Introduction}

The emergence of cloud computing technologies enables clients who are unable to procure and maintain their own computing infrastructure to resort to convenient on-demand computing resources. Despite the paradigm being economically sensible for resource-limited clients, it comes with new security and privacy concerns. One of them is the lack of transparency and control over the outsourced computation, which necessitates the need to verify the result to guarantee integrity of the computation. Another one is the need to protect confidentiality of the data used in the outsourced computation, which can be proprietary, personal, or otherwise sensitive. Addressing these security objectives is thus the focus of this work.

Computation outsourcing to a cloud computing provider is common today and can take different forms. In particular, in addition to the conventional scenario when a computationally-limited client outsources its computation to the cloud and receives the result, there are many uses of cloud computing that involve multiple entities. For example, a doctor's office might send computation associated with a patient's test to a cloud provider, while the patient in question is also entitled to access to the result of the computation and thus should be able to verify integrity of the returned result. This calls for solutions where the integrity of the result can be verified by entities who do not have access to secret keys thus achieving public verifiability. Furthermore, if the task result may be verified by different entities or verified by the same entity multiple times over time (e.g., 
every time the result is used), then it is desirable to lower the overhead associated with verifying the result of the outsourced computation without increasing other costs.

The specific problem that we treat in this work is that of matrix multiplication outsourcing. Because of popularity of matrix multiplication computation in a number of different domains and relatively high cost of this operation on large inputs, secure matrix multiplication outsourcing has received attention in research literature $[4,5,24,39]$. We continue this line of research and show below how our results compare to the state of the art.

A novel feature that we put forward in this work and which is not present in publications that treat the same topic can be described as follows: we divide the overall computation in multiple stages and associate a key with each of them. Only if the computation in the current stage is performed correctly and the correct key is recovered, the server can learn the computation used in the next stage. And without the correct key, it is computationally infeasible to proceed to the next stage and pass verification in any of the stages that follow. This feature allows us to achieve several goals:

1. Chaining of computation from one stage to another allows for more efficient verification of the overall task. That is, corrupting a single cell of the product matrix invalidates the values in all other cells that follow, and verifying the result of the final stage is sufficient in ensuring that the entire task was performed correctly. Other publications (such as $[24,9]$ ), on the other hand, require verification of every matrix cell to ensure correctness of the output.

2. If the server misbehaves during the computation and produces incorrect values for one or more cells of the product matrix, in order to proceed with the computation, it has to invest into substantially larger computation. In other words, the effect of the server's misbehavior is enlarged to the maximum extent, where any deviation from the computation substantially increases the computation cost. Thus, this mechanism is design to deter the server from deviating from the correct computation.

3. When the result is returned to the client and does not pass verification, the client can efficiently identify the first stage during which the server deviated from the prescribed computation and ask the same or different server to rerun the computation starting from that stage.

4. If the server carries out the computation honestly, but gets compromised or infected by malware that corrupts the computation, the server can use the checkpoints between the stages to efficiently determine that corruption took place and quickly recover from it. That is, if the server is unaware of the compromise and continues with the task, the outcome will not pass verification and the computational effort becomes wasted. With the checkpoints, on the other hand, the server will identify the problem, stop the computation, resolve the problem, and resume the task without wasting its efforts.

Our contributions can be summarized as follows:

- We present the core construction for verifiable outsourcing in presence of malicious adversaries who arbitrarily deviates from the prescribed computation (Section 4). In our scheme, delegating a task requires work linear in the input size, carrying out the computation itself has the same complexity as that of conventional matrix multiplication algorithm (i.e., $O\left(n^{3}\right)$ for matrices of dimension $n \times n)$, and verification of the resulting matrix product involves only a single modulo exponentiation and the number of modulo multiplications linear in the output size. 
- We present another construction that assumes rational adversaries who are neither honest nor arbitrarily malicious, but rather economically motivated with the sole purpose of maximizing the monetary reward (Section 5.1). Under this adversarial model, verification of the returned result involves only a single comparison.

- We extend the construction in the rational setting to incorporate the chaining feature as described above without compromising any other properties (Section 5.3). In particular, this has no impact on the complexity of the resulting scheme.

- We next show how data privacy can be added to the constructions in both malicious and rational settings (Section 6). This increases the cost of recovering the output, but it is still linear in the output size.

- Lastly, we extend our constructions to achieve public verifiability, in which any entity with access to public verification key can assess the validity of the returned result (Section 7). When such solutions are combined with data protection, access to the verification key does not allow for recovering the output or input of the computation. The complexities of these solutions also remain low, as in the previous constructions.

All our schemes achieve public delegatability, which means that the entity who runs system setup can be different from the entities who form a task to be outsourced.

In reducing the cost associated with verifiable computation schemes for matrix multiplication outsourcing, our focus was on reducing the cost of verifying the result as this may be a more frequently used operation or an operation performed by weaker clients. The cost of task preparation in our and other schemes requires $O\left(n^{2}\right)$ cryptographic operations for input matrices of size $n \times n$, i.e., linear in the size of input. The server's work for carrying out matrix multiplication is $O\left(n^{3}\right)$ cryptographic operations, i.e., uses the conventional matrix multiplication algorithm.

We note that the cost of $O\left(n^{2}\right)$ cryptographic operations used in task preparation is rather high and will exceed the cost of computing matrix multiplication locally for small matrices. In particular, matrix multiplication algorithms of asymptotic complexity as low as $O\left(n^{2.373}\right)$ are known, but the huge constants hidden behind the big-O notation prevent most of them from being used in practice (i.e., they require more than $2 n^{3}$ work) [3]. In particular, for matrices of dimension $n<10^{20}$, only the algorithm by Strassen 1969 and Winograd 1971 of complexity $O\left(n^{2.807}\right)$ and the technique of trilinear aggregating of complexity $O\left(n^{2.775}\right)$ result in implementations of matrix multiplications that outperform the conventional $O\left(n^{3}\right)$ algorithm $[3,37]$. This means that our and related constructions reduce the cost of matrix multiplication for the client only for large matrices when performing $n^{2}$ cryptographic operations is below $O\left(n^{2.775}\right)$ work.

Before we proceed with the description of our constructions, we discuss related work (Section 2) and provide background information and definitions (Section 3).

\section{Related Work}

We divide all related work in categories and discuss each category in turn.

Verifiable Computation. In verifiable computation $[30,26,22,2,41,40,24,18$, 21], a client outsources a computationally intensive task to a server and verifies its results in an efficient manner upon its completion. The basic question was formulated in work on interactive proofs (IP) [8, 31], efficient arguments based on probabilistically checkable proofs (PCP) $[35,36]$, and computationally sound (CS) proofs [38]. We highlight some of the more relevant results below. 
A typical PCP-based scheme is normally not applicable to our scenario because in such schemes the client needs to maintain a large amount of data in order to verify the results of the computation, while in our setting the client is only capable of storing data linear in the input size. Recently, argument systems based on the work of Kilian [35, 36] have been built [43, 42, 46]. They require the prover to make a commitment to the computation that should be consistent with the queries that will be issued afterwards by the verifier. The client therefore needs to engage in an interactive protocol to carry out the verification procedure. This is also different from our setup, where the client is not involved in interactive computation besides submitting the problem to the server and receiving the results. Parno et al. recently introduced Pinocchio [40], which allows execution of a general function represented as a circuit to be delegated to an untrusted worker. The cost of output verification in [40] is linear in the size of the function's input and output, but requires only a constant number of most expensive operations (pairing evaluation), which resembles similarities to our scheme in presence of malicious adversaries. Our verification cost is still lower in practical terms and is further reduced in the schemes with rational adversaries. The asymptotic complexity of the server's computation in [40] is the same as in our scheme, but our solution offers faster performance. Lastly, the setup of [40] uses a different key generation phase from our problem generation phase, which is applied to a function instead of function's input in our solution. The cost of key generation in [40] is higher than the cost of problem generation in our solution, but the key generation algorithm in [40] may be executed less frequently.

Succinct Non-Interactive Arguments of Knowledge (SNARKs) [11] are CS proofs of knowledge for NP that can potentially be utilized to realize verifiable computation. SNARKs represents a succinct argument system that allows a prover to prove the knowledge of NP statements by performing work independent of the size of the problem instance and its witness. The problem was motivated in early work of Kilian [35] and uses a Merkle hash tree and PCPs to achieve a fourmessage interactive argument for NP. It was later enhanced in [38] to achieve a one-message protocol by additionally incorporating Private Information Retrieval (PIR). As SNARKs are intended for succinct verification using either a designated or public verifier [12], they are considered as an ideal candidate for implementing verifiable computation. However, it is still unknown whether there exists a concrete SNARK construction that relies on standard cryptographic assumptions [29].

Another line of work [26, 22, 21] uses fully homomorphic encryption [28] in combination with verifiable computation to additionally achieve data protection. Unfortunately, these solutions necessitate an expensive offline phase that requires the client to invest $\operatorname{poly}(k, T)$ or $\operatorname{poly}(n, k)$ time, where $T$ is the time it takes to compute the function, $n$ is the memory size, and $k$ is a security parameter, to generate public keys or a commitment used for verification. Our solution does not require any large one-time overhead, which is of benefit to resource-constrained clients who lack sufficient resources, but still would like to delegate the computation.

Homomorphic MAC and Signature Schemes. A homomorphic Message Authentication Code (MAC) scheme [1] allows an entity with possession of a secret key sk to produce a tag $\sigma$ that authenticates message $m$ with a property that allows someone with access to public evaluation key $p v k$ to combine multiple authenticators together. That is, given a set of authenticators $\sigma_{1}, \sigma_{2}, \ldots, \sigma_{n}$ for messages $m_{1}, m_{2}, \ldots, m_{n}$, any entity with $p v k$ is able to homomorphically evaluate a function $P$ on $\left(\sigma_{1}, \sigma_{2}, \ldots, \sigma_{n}\right)$ and produce a short tag $\sigma^{\prime}$ that authenticates correctness of $m^{\prime}=P\left(m_{1}, m_{2}, \ldots, m_{n}\right)$. In the public-key setting, signature schemes are used in place of MAC schemes to result in similar functionality [34]. To realize verifiable computation using a homomorphic MAC (or signature) scheme, a client first sends function $P$ and $\left(m_{i}, \sigma_{i}\right)$ for $1 \leq i \leq n$ as its inputs to a server. The server computes $m^{\prime}=P\left(m_{1}, m_{2}, \ldots, m_{n}\right)$ as a result and the corresponding authenticator $\sigma^{\prime}$ and the returns the values to the client. The client next uses the MAC (or 


\begin{tabular}{|l|c|c|c|c|c|}
\hline \multicolumn{1}{|c|}{ Scheme } & $\begin{array}{c}\text { Verifiable } \\
\text { Computation }\end{array}$ & $\begin{array}{c}\text { Data } \\
\text { Privacy }\end{array}$ & $\begin{array}{c}\text { Public } \\
\text { Verifiability }\end{array}$ & $\begin{array}{c}\text { Output-Indep. } \\
\text { Verification }\end{array}$ & $\begin{array}{c}\text { Deterministic } \\
\text { Verification }\end{array}$ \\
\hline Atallah et al. [4] & $\checkmark$ & $\checkmark$ & $\boldsymbol{X}$ & $\boldsymbol{X}$ & $\boldsymbol{X}$ \\
\hline Mohassel [39] & $\checkmark$ & $\checkmark$ & $\boldsymbol{X}$ & $\boldsymbol{X}$ & $\boldsymbol{X}$ \\
\hline Fiore et al. [24] & $\checkmark$ & $\boldsymbol{X}$ & $\checkmark$ & $\boldsymbol{X}$ & $\checkmark$ \\
\hline Backes et al. [9] & $\checkmark$ & $\boldsymbol{X}$ & $\boldsymbol{X}$ & $\boldsymbol{X}$ & $\checkmark$ \\
\hline This work & $\checkmark$ & $\checkmark$ & $\boldsymbol{\checkmark}$ & $\boldsymbol{\checkmark}$ & $\boldsymbol{\checkmark}$ \\
\hline
\end{tabular}

Table 1: Comparison with related work.

signature) authentication procedure on $\left(m^{\prime}, \sigma^{\prime}\right)$ to verify correctness of computational result $m^{\prime}$. In earlier schemes $[34,14,1,19]$, homomorphic authenticators were mainly used to verify evaluation results of a linear function. They were later extended in $[13,17,27]$ to support more general functions such as constant-degree polynomials and arithmetic circuits with polynomially-bound degrees. In order to apply solutions based on homomorphic authenticators to verifiable computation, the verification mechanism should be much more efficient than executing the function $P$ itself, which is unfortunately not the case for most available schemes. Furthermore, it is not clear as to how to protect secrecy of the underlying messages using these techniques as neither the MAC nor the signature schemes serve this purpose.

Matrix Computation. The problem of verifiable matrix computation has been studied in recent literature $[24,39,4,9]$. In addition to computation verification, existing solutions offer other important security features that are: 1) data protection, i.e., protection of both input and output matrices throughout the computation; 2) public verifiability, i.e., the ability of any entity to verify the result of outsourced computation; and 3) deterministic verification, i.e., the ability to detect faulty cells in an output matrix with probability 1. Unlike prior work, our scheme achieves all of these features. Additionally, we achieve another desirable property called output-independent efficiency that allows for a constant number of cryptographic operations (modulo exponentiations or pairing operations) to be used during verification independent of the size of the output matrix. Table 1 summarizes features of our solution and other schemes. Note that [24] has similar security features to our scheme, and even though not explicitly specified in that work, it is feasible to incorporate privacy protection into their scheme as realized in [47]. We, however, found the scheme cannot be adjusted to efficiently handle rational adversaries.

Next, we provide a more detailed comparison of our work with closely related schemes. Note that we treat deterministic verification as a property that is non-trivial to achieve and thus consider only the work of Fiore et al. [24] and Backes et al. [9]. The construction of [9], however, is based on the scheme of [24] and would offer the same performance as that of [24] in our setting (the advantage of [9] is that it allows for more flexible function specification and scheduling). Thus, we list only performance of [24] as the representative of both [24] and [9].

The computational overhead for the client and the server is presented in Table 2, where $n$ represents the size of each dimension of input matrices, and $c_{m}, c_{e}$, and $c_{p}$ denote the time to carry out a modular multiplication, exponentiation, and a pairing operation, respectively. In the table, we use notation $\mathrm{VC}_{m}$ and $\mathrm{VC}_{r}$ to denote our verifiable computation schemes for malicious and rational adversaries, respectively. Note that some of the constructions for rational adversary do not involve any cryptographic operations for verification (and rather perform a single comparison) and that work is listed as $O(1)$. Finally, note that in some of our constructions for the rational adversary, the work for task preparation or server's computation is increased compared to the equivalent constructions for the malicious adversary, but the verification cost is substantially (and asymptotically) reduced. 


\begin{tabular}{|l|c|c|c|}
\hline \multicolumn{1}{|c|}{ Scheme } & $\begin{array}{c}\text { Client's } \\
\text { Preparation }\end{array}$ & $\begin{array}{c}\text { Server's } \\
\text { Computation }\end{array}$ & $\begin{array}{c}\text { Client's } \\
\text { Verification }\end{array}$ \\
\hline \hline Fiore et al. [24] (priv. ver.) & $\left(4 c_{e}+3 c_{m}\right) n^{2}$ & $c_{e} n^{3}$ & $\left(c_{e}+c_{m}\right) n^{2}$ \\
\hline $\mathrm{VC}_{m}$ (priv. ver.) & $\left(2 c_{e}+4 c_{m}\right) n^{2}$ & $c_{p} n^{3}$ & $c_{e}+c_{m} n^{2}$ \\
\hline $\mathrm{VC}_{r}$ (priv. ver.) & $\left(4 c_{e}+6 c_{m}\right) n^{2}$ & $c_{p} n^{3}+\left(c_{e}+c_{m}\right) n^{2}$ & $O(1)$ \\
\hline \hline Fiore et al. [24] (pub. ver.) & $\left(4 c_{e}+3 c_{m}\right) n^{2}$ & $c_{e} n^{3}$ & $\left(c_{p}+c_{e}+c_{m}\right) n^{2}$ \\
\hline $\mathrm{VC}_{m}$ (pub. ver.) & $\left(2 c_{e}+4 c_{m}\right) n^{2}$ & $c_{e} n^{3}$ & $c_{p} n+\left(c_{e}+c_{m}\right) n^{2}$ \\
\hline $\mathrm{VC}_{r}$ (pub. ver.) & $\left(4 c_{e}+6 c_{m}\right) n^{2}$ & $c_{p} n^{3}+\left(c_{e}+c_{m}\right) n^{2}$ & $c_{e}$ \\
\hline $\mathrm{VC}_{m}$ (priv. ver. + privacy) & $\left(2 c_{e}+6 c_{m}\right) n^{2}$ & $2 c_{p} n^{3}$ & $c_{e}+c_{m} n^{2}$ \\
\hline $\mathrm{VC}_{r}$ (priv. ver. + privacy) & $\left(5 c_{e}+8 c_{m}\right) n^{2}$ & $2 c_{p} n^{3}+c_{m} n^{2}$ & $O(1)$ \\
\hline $\mathrm{VC}_{m}$ (pub. ver. + privacy) & $\left(8 c_{e}+16 c_{m}\right) n^{2}$ & $4 c_{e} n^{3}$ & $4 c_{p} n+4\left(c_{e}+c_{m}\right) n^{2}$ \\
\hline $\mathrm{VC}_{r}$ (pub. ver. + privacy) & $\left(5 c_{e}+8 c_{m}\right) n^{2}$ & $2 c_{p} n^{3}+c_{m} n^{2}$ & $c_{e}$ \\
\hline
\end{tabular}

Table 2: Computation in our and mostly closely related schemes.

\begin{tabular}{|l|c|c|c|}
\hline \multicolumn{1}{|c|}{ Scheme } & Client's Storage & Server's Storage & Communication \\
\hline \hline Fiore et al. [24] (priv. ver.) & $n^{2} \kappa$ & $n^{2} \kappa+2 n^{2}$ & $2 n^{2} \kappa+3 n^{2}$ \\
\hline $\mathrm{VC}_{m}$ (priv. ver.) & $3 n \kappa$ & $2 n^{2} \kappa+2 n^{2}$ & $\left(2 n^{2}+1\right) \kappa+3 n^{2}$ \\
\hline $\mathrm{VC}_{r}$ (priv. ver.) & $\kappa$ & $2 n^{2} \kappa+2 n^{2}$ & $\left(2 n^{2}+1\right) \kappa+3 n^{2}$ \\
\hline \hline Fiore et al. [24] (pub. ver.) & $n^{2} \kappa$ & $n^{2} \kappa+2 n^{2}$ & $2 n^{2} \kappa+3 n^{2}$ \\
\hline $\mathrm{VC}_{m}$ (pub. ver.) & $\left(n^{2}+n\right) \kappa$ & $n^{2} \kappa+2 n^{2}$ & $\left(n^{2}+n\right) \kappa+3 n^{2}$ \\
\hline $\mathrm{VC}_{r}$ (pub. ver.) & $\kappa$ & $2 n^{2} \kappa+2 n^{2}$ & $\left(2 n^{2}+1\right) \kappa+3 n^{2}$ \\
\hline $\mathrm{VC}_{m}$ (priv. ver. + privacy) & $3 n \kappa$ & $4 n^{2} \kappa$ & $\left(5 n^{2}+1\right) \kappa$ \\
\hline $\mathrm{VC}_{r}$ (priv. ver. + privacy) & $\left(n^{2}+2 n\right) \kappa$ & $4 n^{2} \kappa$ & $\left(5 n^{2}+1\right) \kappa$ \\
\hline $\mathrm{VC}_{m}$ (pub. ver. + privacy) & $4\left(n^{2}+n\right) \kappa$ & $4 n^{2} \kappa+8 n^{2}$ & $4\left(n^{2}+n\right) \kappa+12 n^{2}$ \\
\hline $\mathrm{VC}_{r}$ (pub. ver. + privacy) & $\left(n^{2}+2 n\right) \kappa$ & $4 n^{2} \kappa$ & $\left(5 n^{2}+1\right) \kappa$ \\
\hline
\end{tabular}

Table 3: Storage and communication in our and mostly closely related schemes.

The timings of elementary cryptographic operations can be inferred from the benchmarks in [20]. For groups that admit an asymmetric bilinear map $e: \mathbb{G}_{1} \times \mathbb{G}_{2} \rightarrow \mathbb{G}_{T}$, which offer faster performance than groups that admit a symmetric bilinear map $e: \mathbb{G}_{1} \times \mathbb{G}_{1} \rightarrow \mathbb{G}_{T}$ and are used in this work (as detailed later in the paper) and can be used in the solution of [24], the timings are as follows: for 128-bit security, a modulo exponentiation in $\mathbb{G}_{1}$ or $\mathbb{G}_{2}$ can be performed in $0.1-0.4 \mathrm{~ms}$ on a single core of a conventional $2.4 \mathrm{GHz}$ desktop machine, a modulo exponentiation in $\mathbb{G}_{T}$ in $0.4-0.9 \mathrm{~ms}$, and the pairing operation in $2.1-2.3 \mathrm{~ms}$. The cost of modulo multiplications is at least two orders of magnitude smaller than that of performing modulo exponentiations.

The storage and communication requirements of our constructions and those of [24] are listed in Table 3. In the table, the security parameter $\kappa$ denotes the bitlength of group elements. The client's storage is computed as the amount of information the client needs to maintain in order to be able to verify the result (i.e., the size of the key). Then the task delegator and each task verifier will require additional storage for their respective input and output matrices. The server's storage corresponds to the amount of storage the server needs to maintain in order to carry out the task. Lastly, communication corresponds to both sending the task to the server and returning the result and the proof of computation to the client.

Rational Computation. In recent years, game theory has been used in cryptographic research [23, 33 to develop a new adversarial model - a rational adversary who is no longer treated as arbitrarily malicious, but who is motivated by some utility function with the sole purpose of maximizing its utility. It is known that under this model protocols can be designed with better efficiency than that of traditional counterparts [25]. As our problem deals with verifiable computation, we are interested 
in rational proof systems that have been recently studied in $[6,7,32]$. The essential merits of rational proof systems are that they allow for extremely low communication and verification time [6,7] and can even achieve single-round proofs if the prover is computationally bounded [32]. The basic idea of this line of work is that the prover will send the result of computation to the verifier who will compute the corresponding reward based on the "quality" of prover's result, and the reward will be maximized only if the result is correct. The publications focus on general complexity classes such as uniform $T C^{0}$ (polynomial-time, constant-depth threshold circuits) and decision problems in $P^{\| N P}$ (polynomial time with access to parallel queries to an NP oracle), while in our case, we target specific matrix computation with the goal of achieving even better efficiency.

Another related work in this setting is the hourglass scheme [45] which is a technique employed by a tenant for enforcing an economically rational cloud provider to keep the user's data at rest encrypted (even when it possesses decryption keys). The basic idea is to encode the data after it has been encrypted in such a way that applying the encoding on the fly upon receiving the tenant's challenges imposes significant resource constraints on the provider. Therefore, by observing the time it takes to serve the tenant's request, the tenant will know whether the provider is complying with the storage policy. Note that the scheme does not work in presence of fully malicious adversaries (who, e.g., can store both plaintext files and their encoded versions) and this is why the approach only targets economically rational adversaries who adopt the most reasonable strategy that maximizes their profits. Our work applies a similar idea to computation of matrix multiplication instead of storage (i.e., if the provider deviates from the prescribed computation, it will have to invest larger computation into passing verification).

\section{Background and Definitions}

\subsection{Basic Definitions}

Throughout this work we use notation $x \stackrel{R}{\leftarrow} S$ to denote that $x$ is chosen uniformly at random from the set $S$. A function $\epsilon(n)$ is said to be negligible if for sufficiently large $n$ its value is smaller than the inverse of any polynomial poly $(n)$. Let $F$ be a family of functions and $\operatorname{Dom}(f)$ denote the domain of function $f \in F$. Also, let $\kappa$ denote a security parameter. We use $H:\{0,1\}^{*} \rightarrow\{0,1\}^{\ell_{1}(\kappa)}$ to denote a collision resistant hash function that takes as input a string $x$ and outputs an $\ell_{1}(\kappa)$-bit hash $y$. We also use notation $\|$ to denote string concatenation. For matrix $A$, notation $A_{i j}$ refers to the element of $A$ at row $i$ and column $j$. We use notation PRF to refer to a pseudo-random function family.

Definition 1 Let $F:\{0,1\}^{\kappa} \times\{0,1\}^{\ell_{2}(\kappa)} \rightarrow\{0,1\}^{\ell_{2}(\kappa)}$ be a family of functions. For $k \in\{0,1\}^{\kappa}$, the function $f_{k}:\{0,1\}^{\ell_{2}(\kappa)} \rightarrow\{0,1\}^{\ell_{2}(\kappa)}$ is defined as $f_{k}(x)=F(k, x)$. F is said to be a family of pseudo-random functions (PRF) if for every probabilistic polynomial time (PPT) adversary $\mathcal{A}$ with oracle access to a function $f_{k}$ and all sufficiently large $\kappa, \mid \operatorname{Pr}\left[\mathcal{A}^{f_{k}}\left(1^{\kappa}\right)-\operatorname{Pr}\left[\mathcal{A}^{R}\left(1^{\kappa}\right)\right] \mid\right.$ is negligible in $\kappa$, where $k \stackrel{R}{\leftarrow}\{0,1\}^{\kappa}$ and $R$ is a function chosen at random from all possible functions mapping $\ell_{2}(\kappa)$-bit inputs to $\ell_{2}(\kappa)$-bit outputs.

Definition 2 (Bilinear map) A one-way function $e: \mathbb{G}_{1} \times \mathbb{G}_{2} \rightarrow \mathbb{G}_{T}$ is a bilinear map if the following conditions hold:

- (Efficient) $\mathbb{G}_{1}, \mathbb{G}_{2}$, and $\mathbb{G}_{T}$ are groups of the same prime order $p$ and there exists an efficient algorithm for computing e.

- (Bilinear) For all $g_{1} \in \mathbb{G}_{1}, g_{2} \in \mathbb{G}_{2}$, and $a, b \in \mathbb{Z}_{p}, e\left(g_{1}^{a}, g_{2}^{b}\right)=e\left(g_{1}, g_{2}\right)^{a b}$. 
- (Non-degenerate) If $g_{1}$ generates $\mathbb{G}_{1}$ and $g_{2}$ generates $\mathbb{G}_{2}$, then e $\left(g_{1}, g_{2}\right)$ generates $\mathbb{G}_{T}$.

Throughout this work, we assume there exists a trusted setup algorithm Set that, on input a security parameter $1^{\kappa}$, outputs the setup for groups $\mathbb{G}_{1}=\left\langle g_{1}\right\rangle$ and $\mathbb{G}_{2}=\left\langle g_{2}\right\rangle$ of prime order $p$ that have a bilinear map $e$, and $e\left(g_{1}, g_{2}\right)$ generates $\mathbb{G}_{T}$ of order $p$. That is, $\left(p, \mathbb{G}_{1}, \mathbb{G}_{2}, \mathbb{G}_{T}, g_{1}, g_{2}, e\right) \leftarrow \operatorname{Set}\left(1^{\kappa}\right)$.

\subsection{Computational Assumptions}

The first computational assumption that we use in this work is the Multiple Decisional DiffieHellman Assumption ( $m$-M-DDH) [16], which can be stated as follows:

Definition 3 ( $m$-M-DDH assumption) Let $\mathbb{G}$ be a group of prime order $p, g \in \mathbb{G}$ is its generator, and $m \geq 2$. Also let $D=\left(g^{x_{1}}, \ldots, g^{x_{m}},\left\{g^{x_{i} x_{j}}\right\}_{1 \leq i<j \leq m}\right)$ for random $x_{1}, \ldots, x_{m} \in \mathbb{Z}_{p}$, and define random tuple as $D_{\text {rand }}=\left(g_{1}, \ldots, g_{m},\left\{g_{i j}\right\}_{1 \leq i<j \leq m}\right)$ in $\mathbb{G}$. Adversary $\mathcal{A}$, whose task is to distinguish an $M-D D H$ tuple from a random tuple, outputs a bit. We define the advantage of adversary $\mathcal{A}$ in solving the $M-D D H$ problem as $\boldsymbol{A} \boldsymbol{d v}_{\mathcal{A}}^{\mathrm{M}-\mathrm{DDH}}(\kappa)=\mid \operatorname{Pr}[\mathcal{A}(g, p, m, D)=1]-\operatorname{Pr}\left[\mathcal{A}\left(g, p, m, D_{\text {rand }}\right)=\right.$ 1]|. The $M-D D H$ assumption holds if for every PPT algorithm $\mathcal{A}, \boldsymbol{A} \boldsymbol{d} \boldsymbol{v}_{\mathcal{A}}^{\mathrm{M}-\mathrm{DDH}}(\kappa)$ is negligible.

Some of our schemes are built using subgroups of elliptic curves with pairings where the decisional Diffie-Hellman (DDH) problem is hard. The use of DDH-hard pairing groups requires the External Diffie-Hellman (XDH) assumption [10].

Definition 4 (XDH assumption) Let $\left(p, \mathbb{G}_{1}, \mathbb{G}_{2}, \mathbb{G}_{T}, g_{1}, g_{2}, e\right) \leftarrow \operatorname{Set}\left(1^{\kappa}\right)$. We define the advantage of adversary $\mathcal{A}$ in solving the DDH problem in $\mathbb{G}_{1}$ as

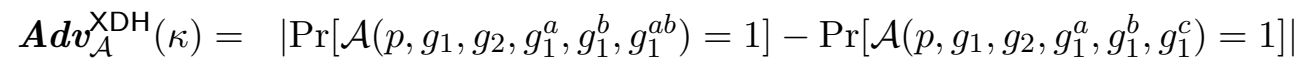

where $a, b, c \stackrel{R}{\leftarrow} \mathbb{Z}_{p}$. We say that the XDH assumption holds if for every PPT algorithm $\mathcal{A}$ $\boldsymbol{A} \boldsymbol{d} \boldsymbol{v}_{\mathcal{A}}^{\mathrm{XDH}}(\kappa)$ is negligible.

The XDH assumption implies that there is no efficiently computable homomorphism from $\mathbb{G}_{1}$ to $\mathbb{G}_{2}$. This assumption is also necessary for the M-DDH assumption to hold in groups that admit a bilinear map.

\subsection{Verifiable Computation}

A verifiable computation scheme VC is a 4-tuple of polynomial-time algorithms (Setup, ProbGen, Compute, Verify) that allows a user to outsource the computation of function $f \in F$ to an untrusted worker. VC is defined as follows:

$\operatorname{Setup}\left(1^{\kappa}, f\right) \rightarrow$ params: On input a security parameter $\kappa$ and function $f$ to be outsourced, it produces public parameters params.

$\operatorname{ProbGen}(x$, params $) \rightarrow\left(\mathrm{SK}_{x}, \mathrm{EK}_{x}, \sigma_{x}\right)$ : Given an input $x \in \operatorname{Dom}(f)$, this algorithm is run by the delegator to produce a secret key $\mathrm{SK}_{x}$ associated with the problem instance for computation outsourcing and output verification, an evaluation key $\mathrm{EK}_{x}$ given to the worker to carry out the outsourced computation, and an encoding $\sigma_{x}$ of input $x$.

Compute $\left(\mathrm{EK}_{x}, \sigma_{x}\right) \rightarrow \sigma_{y}$ : On an encoded input $\sigma_{x}$ and $\mathrm{EK}_{x}$, the worker runs the algorithm to produce an encoded outcome $\sigma_{y}$, where $y=f(x)$.

Verify $\left(\mathrm{SK}_{x}, \sigma_{y}\right) \rightarrow y \cup \perp$ : Given an encoded output $\sigma_{y}$ and the secret key $\mathrm{SK}_{x}$, this algorithm outputs $y$ or an error $\perp$ upon result verification. 
The correctness requirement is such that the values produced by the algorithms will allow any honest worker who faithfully executes Compute to pass verification of the output it produces. More formally, for any $f \in F$, any params $\leftarrow \operatorname{Setup}\left(1^{\kappa}, f\right)$, and any $x \in \operatorname{Dom}(f)$, if $\left(\mathrm{SK}_{x}, \mathrm{EK}_{x}, \sigma_{x}\right) \leftarrow$ $\operatorname{ProbGen}(x$, params $), \sigma_{y} \leftarrow \operatorname{Compute}\left(\mathrm{EK}_{x}, \sigma_{x}\right)$, and $y \leftarrow \operatorname{Verify}\left(\mathrm{SK}_{x}, \sigma_{y}\right)$, then $\operatorname{Pr}[y=f(x)]=1$.

To formulate security of a verifiable computation scheme, we define an interactive security experiment described next. In the experiment, the adversary $\mathcal{A}$ is allowed to query ProbGen algorithms on inputs of its choice $x_{i}$ and obtain the corresponding evaluation key $\mathrm{EK}_{x_{i}}$ and input encoding $\sigma_{x_{i}}$. In the private key setting, it is also granted oracle access to Verify algorithm, where $\mathcal{O}_{\text {Verify }}\left(x_{i}, \sigma_{y}\right)$ runs $y \leftarrow \operatorname{Verify}\left(\mathrm{SK}_{i}, \sigma_{y}\right)$ and returns $y$. Eventually, $\mathcal{A}$ outputs the input $x^{*}$ on which it would like to be challenged, obtains evaluation key $\mathrm{EK}_{x^{*}}$ and encoding $\sigma_{x^{*}}$, and produces output encoding $\sigma_{y}^{\prime}$. The adversary succeeds if the output is different from $f\left(x^{*}\right)$ and the verification algorithm does not output an error $\perp$. Note that this definition captures full adaptive security as opposed to weaker selective security where the adversary is required to commit to the challenge input $x^{*}$ in the beginning of the game.

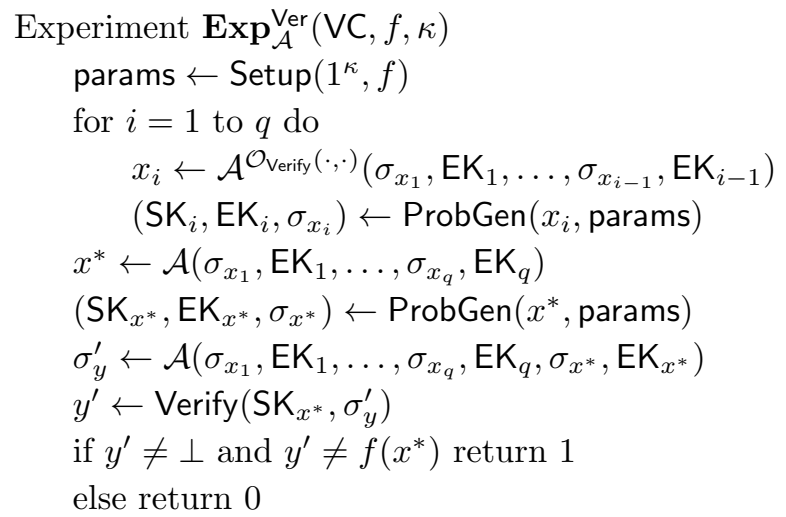

For any $\kappa \in \mathbb{N}$ and any function $f \in F$, we define the advantage of an adversary $\mathcal{A}$ making at most $q=\operatorname{poly}(\kappa)$ queries in the above security game against $\mathrm{VC}$ as $\mathbf{A d v}_{\mathcal{A}}^{\operatorname{Ver}}(\mathrm{VC}, f, q, \kappa)=$ $\operatorname{Pr}\left[\operatorname{Exp}_{\mathcal{A}}^{\operatorname{Ver}}(\mathrm{VC}, f, \kappa)=1\right]$.

Definition 5 A verifiable computation scheme VC is secure if for any PPT adversary $\mathcal{A}$, any $\kappa$, and any $f \in F, \boldsymbol{A} \boldsymbol{d} \boldsymbol{v}_{\mathcal{A}}^{\mathrm{Ver}}(\mathrm{VC}, f, q, \kappa)$ is negligible in $\kappa$.

In this work we consider two types of adversaries: the first type is the traditional adversary that can arbitrarily deviate from the prescribed computation as defined by Compute functionality. We denote this type of adversary as malicious. While the malicious adversary model leads to strong security guarantees, it has been criticized as overly pessimistic due to neglecting the incentive that could potentially cause computational entities to deviate from the prescribed behavior. We therefore consider the second type of adversary which we denote as rational. A rational adversary is neither honest nor malicious, but only interested in maximizing its reward attained during computation. The rationale behind including this type of adversary is that it allows us to design more efficient solutions if the server can be assumed not to intentionally corrupt the result. Next, we formally define the rational adversary model in verifiable computation, which was initially proposed in [6] and later refined for rational argument systems in $[7,32]$.

Definition 6 A function $f$ admits a rational argument with security parameter $\kappa$ if there exists a protocol $(P, V)$ and a randomized reward function reward: $\{0,1\}^{\star} \rightarrow \mathbb{R}_{\geq 0}$ such that for any prover $\hat{P}$ of size $\leq 2^{\kappa(|x|)}$ and input $x \in\{0,1\}^{\star}$, the following three properties hold:

- $\operatorname{Pr}[$ output $(P, V)(x)=f(x)]=1$.

- There exists a negligible function $\mu(\cdot)$ such that $E[\operatorname{reward}((P, V)(x))]+\mu(|x|) \geq E[\operatorname{reward}((\hat{P}, V)(x))]$. 
- If there exists a polynomial $p(\cdot)$ such that $\operatorname{Pr}[$ output $((\hat{P}, V)(x)) \neq f(x)] \geq p(|x|)^{-1}$, then there exists a polynomial $q(\cdot)$ such that $E[\operatorname{reward}((P, V)(x))] \geq E[\operatorname{reward}((\hat{P}, V)(x))]+q(|x|)^{-1}$.

The first property refers to completeness of the protocol, which says prover $P$ is able to return a correct answer $f(x)$ by following the prescribed protocol. The second property ensures that by deviating from the prescribed protocol in a computationally bounded manner, a dishonest prover $\hat{P}$ will achieve at most negligibly larger gain than a faithful prover $P$. The last property guarantees if $\hat{P}$ does not report a correct answer $f(x)$ with a noticeable probability, he has to bear a noticeable utility loss. A rational argument system ensures that a rational adversary will maximize the reward if and only if he honestly follows the protocol to report the correct answer. Therefore, to prove security of a protocol, we need to show that it conforms to Definition 6 under reasonable assumptions on cost and utility, which we formulate in the server-client setting as follows:

Assumption 1 - For each outsourced task, both the monetary reward the client compensates and the computational cost the server bears are polynomial to the size of the input.

- As the server aims to make profits by devoting his resources to clients' specific tasks, the monetary reward he gains from a client should be larger than the cost it bears, which also conforms to the business practice for cloud service.

- In the event of any inconsistency between the answers the server returns and the answers the client expects, the server will not receive any reward or even undertake utility loss resulted from the violation of Service Level Agreement.

\subsection{Data Protection in Outsourced Computation}

When the data used in an outsourced task is sensitive (e.g., private, proprietary, etc.), it is desirable to additionally guarantee secrecy of the data. To formulate secrecy protection of data used in verifiable computation, we define an interactive security experiment described next. In the experiment, the adversary $\mathcal{A}$ is allowed to query ProbGen on inputs $x_{i}$ of its choice a polynomial number of times, obtain the corresponding evaluation keys $\mathrm{EK}_{x_{i}}$ and the encoded inputs $\sigma_{x_{i}}$, and carry out arbitrary computations including Compute that produces $\sigma_{y_{i}}$. Eventually, $\mathcal{A}$ outputs two strings $x^{(1)}$ and $x^{(2)}$ on which it would like to be challenged. A random bit $b$ is drawn. $\mathcal{A}$ is given encoding of $x^{(b)} \sigma_{x^{(b)}}$ together with the evaluation key $\mathrm{EK}_{x^{(b)}}$ produced using ProbGen. The adversary $\mathcal{A}$ outputs its guess for $b$ and wins if it could correctly determine the value of $b$, i.e., distinguish between inputs $x^{(0)}$ and $x^{(1)}$.

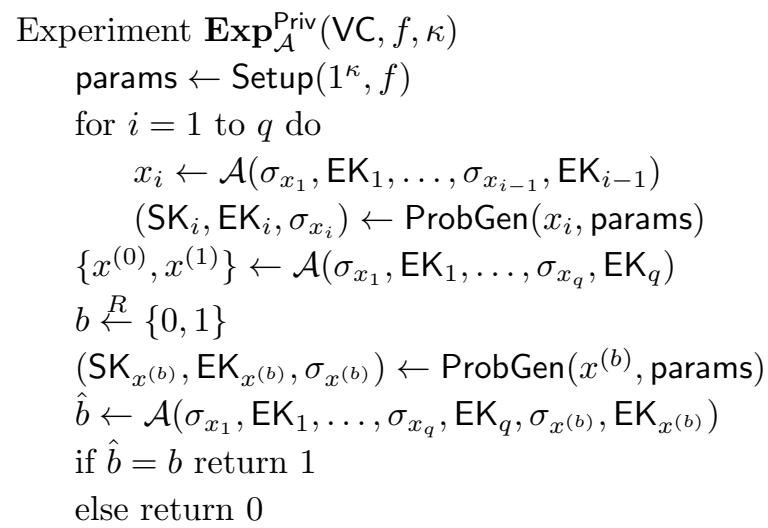

For any $\kappa \in \mathbb{N}$ and any function $f \in F$, we define the advantage of an adversary $\mathcal{A}$ making at most $q=\operatorname{poly}(\kappa)$ queries in the above security game against $\mathrm{VC}$ as

$$
\mathbf{A d v}_{\mathcal{A}}^{\text {Priv }}(\mathrm{VC}, f, q, \kappa)=\operatorname{Pr}\left[\mathbf{E x p}_{\mathcal{A}}^{\text {Priv }}(\mathrm{VC}, f, \kappa)=1\right]-1 / 2
$$


Definition 7 We say that a verifiable computation scheme VC ensures data secrecy if for any PPT adversary $\mathcal{A}$, any $\kappa$, and any $f \in F$, it holds that the advantage $\boldsymbol{A d v}_{\mathcal{A}}^{\text {Priv }}(\mathrm{VC}, f, q, \kappa)$ is negligible.

When we talk about verifiable computation schemes that provide data protection, we separate the algorithms for computation verification and output recovery. In particular, Verify now only assesses the validity of the returned (protected) output, while new algorithm Output recovers the data itself. More formally, we have:

Verify $\left(\mathrm{SK}_{x}, \sigma_{y}\right) \rightarrow b$ : Given an encoded output $\sigma_{y}$ and the secret key $\mathrm{SK}_{x}$, this algorithm outputs a bit $b$ which is set to 1 iff verification was successful.

Output $\left(\mathrm{SK}_{x}, \sigma_{y}\right) \rightarrow y \cup \perp$ : Given an encoded output $\sigma_{y}$ and the secret key $\mathbf{S K}_{x}$, this algorithm returns the output $y$ or $\perp$ if the output could not be recovered.

This separation is particularly useful for solutions with public verifiability (where the verifier may not have the ability to recover the output) as described next.

\subsection{Public Verifiability}

Recall that public verifiability allows any entity with access to public verification key to assess correctness of the result of outsourced computation. To model this formally, we modify the ProbGen algorithm to produce public verification key, denoted by $\mathrm{PVK}_{x}$, and have Verify take PVK instead of $\mathrm{SK}_{x}$ as before. More precisely, the interface for ProbGen is now

$$
\operatorname{ProbGen}(x, \text { params }) \rightarrow\left(\mathrm{SK}_{x}, \mathrm{PVK}_{x}, \mathrm{EK}_{x}, \sigma_{x}\right),
$$

and the interface for Verify is either

$$
\operatorname{Verify}\left(\mathrm{PVK}_{x}, \sigma_{y}\right) \rightarrow y \cup \perp \quad \text { or } \quad \operatorname{Verify}\left(\mathrm{PVK}_{x}, \sigma_{y}\right) \rightarrow b
$$

without and with data protection, respectively. Note that ProbGen may still produce $\mathrm{SK}_{x}$, which in particular is used to recover the output in schemes that support data protection.

Incorporating public verifiability into a verifiable computation scheme affects the security experiment in which the adversary participates, and we denote the new security experiment by $\operatorname{Exp}_{\mathcal{A}}^{\text {PubVer }}(\mathrm{VC}, f, \kappa)$. The difference from the original definition is that now the adversary is given public verification keys corresponding to all problems that it queried. For completeness, the full modified definition is given in Appendix A. As before, the advantage of an adversary $\mathcal{A}$ making at most $q=\operatorname{poly}(\kappa)$ queries in the security experiment against some scheme VC is defined as

$$
\mathbf{A d v}_{\mathcal{A}}^{\text {PubVer }}(\mathrm{VC}, f, q, \kappa)=\operatorname{Pr}\left[\mathbf{E x p}_{\mathcal{A}}^{\text {PubVer }}(\mathrm{VC}, f, \kappa)=1\right]
$$

We then obtain the following modified security definition:

Definition 8 We say that a verifiable computation scheme with public verifiability VC is secure if for any PPT adversary $\mathcal{A}$, any $\kappa$, and any $f \in F$, it holds that $\boldsymbol{A d v}_{\mathcal{A}}^{\mathrm{PubVer}}(\mathrm{VC}, f, q, \kappa)$ is negligible in $\kappa$.

\section{Matrix Multiplication in Presence of Malicious Adversary}

\subsection{Problem Formulation}

The delegator would like to multiply two matrices $A$ and $B$ of dimensions $n_{1} \times n_{2}$ and $n_{2} \times n_{3}$, respectively. It is assumed that the elements of $A$ and $B$ are not sensitive and do not require 
protection. In our solution, the delegator's work is linear in the size of the input and output, which is optimal.

Our first scheme aims to defend against a malicious adversary who tampers with the computation or its results regardless of operation costs and attempts to pass the verification test. Also, this basic construction does not achieve public verifiability: only the entity who possesses the secret key is able to attest the correctness of outsourced computation. In a consecutive solution in section 7 we extend the scheme to support public verifiability that allows any entity with access to public key assess the validity of computation results. For notational simplicity, we use $\mathrm{VC}_{m}$ to denote this scheme.

\subsection{Description of the Scheme}

The main idea used in our solution is that the delegator encodes matrix $A$ into matrix $X$ and matrix $B$ into matrix $Y$. The delegator sends matrices $\{A, B, X, Y\}$ to the server who computes $C=A \times B$ and $D=X \times Y$. The client then verifies correctness of $C$ by checking a secret relationship between the elements of $C$ and $D$.

In more detail, the secret relationship is formed by generating secret random group elements $R_{i j}$ such that $D_{i j}=R_{i j}^{C_{i j}}$, which are of the form $g_{T}^{r_{i} c_{j}}$, where $\left\{r_{i}\right\}_{i=1}^{n_{1}}$ and $\left\{c_{j}\right\}_{j=1}^{n_{3}}$ are two vectors of random elements. Moreover, in order to satisfy the relationship, we need to embed $r_{i}$ and $c_{j}$ into each $D_{i j}$, and this is realized by forming $X_{i j}$ and $Y_{i j}$ to be of the form $g_{1}^{r_{i} A_{i j}}$ and $g_{2}^{c_{j} B_{i j}}$, respectively. We aim to rely on the M-DDH assumption to show that, given $g_{1}^{r_{i}}$ and $g_{2}^{c_{j}}, g_{T}^{r_{i} c_{j}}$ is indistinguishable from a random value, which is however difficult due to the existence of bilinear pairing operation. To remedy the problem, we completely hide all information about $g_{2}^{c_{j}}$ 's from the server. As a result, if we rely on the XDH assumption, $\left(g_{T}^{r_{1}}, \ldots, g_{T}^{r_{n_{1}}},\left\{g_{T}^{r_{i} c_{j}}\right\}_{1 \leq i \leq n_{1}, 1 \leq j \leq n_{3}}\right)$ is a partial $\left(n_{1}+n_{3}\right)$-M-DDH tuple and the adversary can have only a negligible advantage in distinguishing the $g_{T}{ }^{r_{i} c_{j}}$ 's from random elements of the group. The hiding is achieved by further masking each $Y_{i j}$ by a random value $T_{i j}$, which should be also in a special form; otherwise, the client will have to compute $X \times T$ to satisfy the relationship, which is the exact workload the client wants to avoid. Therefore, $T_{i j}$ 's

are formed as $g_{2}^{w_{j} d_{i}}$ using two random vectors $\left\{d_{i}\right\}_{i=1}^{n_{2}}$ and $\left\{w_{j}\right\}_{j=1}^{n_{3}}$, and the client only needs to perform $O\left(n_{1} n_{3}\right)$ work to compute $X \times T$.

When the server returns $C$ and $\sum_{i} \sum_{j} D_{i j}$, the delegator uses $\left\{r_{i} c_{j}\right\}_{1 \leq i \leq n_{1}, 1 \leq j \leq n_{3}}$ and information about the product $X \times T$ to verify that the sum of the elements in $C$ after proper randomization matches $\sum_{i} \sum_{j} D_{i j}$. If the verification succeeds, the delegator uses $C$ as the correct output. The details are given in Figure 1.

The complexity of ProbGen run by the delegator is dominated by computing key $v k$ and matrices $X$ and $Y$, and is therefore $O\left(n_{1} n_{2}+n_{2} n_{3}\right)$. Compute involves the execution of two matrix multiplications resulting in complexity $O\left(n_{1} n_{2} n_{3}\right)$. Verify consists of ensuring the validity of $C$ by checking its elements against $s$ that Compute produces and has complexity $O\left(n_{1} n_{3}\right)$, i.e., linear in the size of the output. The value of $s$ is computed in such a way that a malicious adversary is unable to construct an incorrect $s$ that passes the verification test.

\subsection{Analysis of the Scheme}

To demonstrate correctness, we show that if the computation was performed correctly, Verify outputs product $A \times B$. In Verify, we have:

$$
g_{T}^{\sum_{i=1}^{n_{1}} r_{i} \sum_{j=1}^{n_{3}} c_{j} C_{i j}+v k_{i}}=\prod_{i=1}^{n_{1}} g_{T}^{r_{i} \sum_{j=1}^{n_{3}} c_{j} C_{i j}+v k_{i}}
$$


Setup $\left(1^{\kappa}, f\right)$ : Given $f$ that indicates matrix multiplication, using the security parameter $\kappa$ run $\left(p, \mathbb{G}_{1}, \mathbb{G}_{2}, \mathbb{G}_{T}, g_{1}, g_{2}, e\right) \leftarrow \operatorname{Set}\left(1^{\kappa}\right)$ and set Set params $=\left(p, \mathbb{G}_{1}, \mathbb{G}_{2}, \mathbb{G}_{T}, g_{1}, g_{2}, g_{T}=\right.$ $\left.e\left(g_{1}, g_{2}\right), f\right)$. Matrix elements should be representable as values in $\mathbb{Z}_{p}$.

$\operatorname{ProbGen}\left(x=(A, B)\right.$, params): On input two matrices $A$ and $B$ of respective dimensions $n_{1} \times n_{2}$ and $n_{2} \times n_{3}$, perform:

1. Choose $r_{i} \stackrel{R}{\leftarrow} \mathbb{Z}_{p}^{*}$ for $1 \leq i \leq n_{1}, d_{j} \stackrel{R}{\leftarrow} \mathbb{Z}_{p}^{*}$ for $1 \leq j \leq n_{2}$, and $c_{k}, w_{k} \stackrel{R}{\leftarrow} \mathbb{Z}_{p}^{*}$ for $1 \leq k \leq n_{3}$.

2. Compute $t_{i}=\sum_{k=1}^{n_{2}} A_{i k} d_{k}$ for $1 \leq i \leq n_{1}, w=\sum_{j=1}^{n_{3}} w_{j}$, and $v k_{i}=r_{i} t_{i} w$ for $1 \leq i \leq$ $n_{1}$.

3. Compute $X_{i j}=g_{1}^{r_{i} A_{i j}}$ for $1 \leq i \leq n_{1}$ and $1 \leq j \leq n_{2}$.

4. Compute $Y_{i j}=g_{2}^{c_{j} B_{i j}+w_{j} d_{i}}$ for $1 \leq i \leq n_{2}$ and $1 \leq j \leq n_{3}$.

5. Set $\mathrm{SK}_{x}=\left(\left\{c_{i}\right\}_{i=1}^{n_{3}},\left\{v k_{i}\right\}_{i=1}^{n_{1}},\left\{r_{i}\right\}_{i=1}^{n_{1}}\right), \mathrm{EK}_{x}=$ params, and $\sigma_{x}=(A, B, X, Y)$.

Compute $\left(\mathrm{EK}_{x}, \sigma_{x}=(A, B, X, Y)\right)$ : Given $\sigma_{x}$, execute:

1. Compute $C=A \times B$.

2. Compute $s=\prod_{i=1}^{n_{1}} \prod_{j=1}^{n_{3}} \prod_{k=1}^{n_{2}} e\left(X_{i k}, Y_{k j}\right)$.

3. Set $\sigma_{y}=(C, s)$.

$\operatorname{Verify}\left(\mathrm{SK}_{x}=\left(\left\{c_{i}\right\}_{i=1}^{n_{3}},\left\{v k_{i}\right\}_{i=1}^{n_{1}},\left\{r_{i}\right\}_{i=1}^{n_{1}}\right), \sigma_{y}=(C, s)\right)$ : Verify whether $g_{T}^{\sum_{i=1}^{n_{1}} r_{i} \sum_{j=1}^{n_{3}} c_{j} C_{i j}+v k_{i}}=s$. If the check succeeds, output $C$; otherwise, output $\perp$.

Figure 1: Description of the core scheme $\mathrm{VC}_{m}$ in presence of malicious adversaries.

$$
\begin{aligned}
& =\prod_{i=1}^{n_{1}} \prod_{j=1}^{n_{3}} g_{T}^{r_{i} c_{j} C_{i j}+r_{i} t_{i} w_{j}}=\prod_{i=1}^{n_{1}} \prod_{j=1}^{n_{3}} g_{T}^{\sum_{k=1}^{n_{2}} r_{i} A_{i k}\left(c_{j} B_{k j}+w_{j} d_{k}\right)} \\
& =\prod_{i=1}^{n_{1}} \prod_{j=1}^{n_{3}} \prod_{k=1}^{n_{2}} e\left(g_{1}^{r_{i} A_{i k}}, g_{2}^{c_{j} B_{k j}+w_{j} d_{k}}\right)=\prod_{i=1}^{n_{1}} \prod_{j=1}^{n_{3}} \prod_{k=1}^{n_{2}} e\left(X_{i k}, Y_{k j}\right) \\
& =s
\end{aligned}
$$

The security property that this scheme achieves can be stated as follows:

Theorem 1 Assuming that the $M-D D H$ and $X D H$ problems are hard, the verifiable computation scheme $\mathrm{VC}_{m}$ is secure according to Definition 5 in presence of malicious adversaries.

Theorem 1 Our proof follows the hybrid argument. We start with the security experiment $\operatorname{Exp}_{\mathcal{A}} \operatorname{Ver}\left(\mathrm{VC}_{m}, f, \kappa\right)$ and devise a sequence of security games, where the adversary $\mathcal{A}$ 's view in one game is indistinguishable from its view in another game. We analyze $\mathcal{A}$ 's advantage $\mathbf{A d} \mathbf{d v}_{\mathcal{A}} \mathrm{Ver}\left(\mathrm{VC}_{m}, f, q, \kappa\right)$ in winning the experiment. Let $T_{i}$ denote the event that the security experiment returns 1 in game $\mathbf{G}_{\mathbf{i}}$. The security games are defined as follows:

Game $\mathbf{G}_{\mathbf{0}}$. Define $\mathbf{G}_{\mathbf{0}}$ to be the same as $\operatorname{Exp}_{\mathcal{A}}^{\operatorname{Ver}}\left(\mathrm{VC}_{m}, f, \kappa\right)$.

Game $\mathbf{G}_{\mathbf{1}}$. The game is identical to $\mathbf{G}_{\mathbf{0}}$, except that when generating $Y_{i j}$, the delegator will use random value $r_{i j}^{(1)}$ in $\mathbb{Z}_{p}$ instead of $w_{j} d_{i}$. In other words, each $Y_{i j}$ is formed as $g_{2}{ }^{c_{j} B_{i j}+r_{i j}^{(1)}}$ as opposed to $g_{2}^{c_{j} B_{i j}+w_{j} d_{i}}$ in game $\mathbf{G}_{\mathbf{0}}$. To be able to verify the result of computation, the delegator also changes each $v k_{i}$ in $\mathrm{SK}_{x}$ from its original value $r_{i} \sum_{k=1}^{n_{2}} \sum_{j=1}^{n_{3}} A_{i k} d_{k} w_{j}$ to $r_{i} \sum_{k=1}^{n_{2}} \sum_{j=1}^{n_{3}} A_{i k} r_{k j}^{(1)}$, 
while keeping the remaining portion of $\mathrm{VC}_{m}$ construction unchanged. This will allow the delegator to verify the result of computation without any changes to Verify.

Comparing $\mathcal{A}$ 's view in games $\mathbf{G}_{\mathbf{0}}$ and $\mathbf{G}_{\mathbf{1}}$, we have that $g_{1}^{w_{j}} d_{i}$, s are replaced with random group elements. Now notice that all $g_{1}^{w_{j} d_{i}}$, s collectively form a partial $\left(n_{2}+n_{3}\right)$-M-DDH tuple. This gives us that the advantage $\mathcal{A}$ has in game $\mathbf{G}_{\mathbf{0}}$ is at most $\mathbf{A} \mathbf{d} \mathbf{v}_{\mathcal{A}}^{\mathrm{M}-\mathrm{DDH}}(\kappa)$ larger than in game $\mathbf{G}_{\mathbf{1}}$ and thus any non-negligible difference in the adversary's behavior between the games $\mathbf{G}_{\mathbf{0}}$ and $\mathbf{G}_{\mathbf{1}}$ can be used to break the M-DDH assumption. Therefore, we have that $\left|\operatorname{Pr}\left[T_{1}\right]-\operatorname{Pr}\left[T_{0}\right]\right| \leq \mathbf{A d v}_{\mathcal{A}}^{\mathrm{M}-\mathrm{DDH}}(\kappa)$ and based on our assumption that the M-DDH problem is hard the difference in the adversary's view between games $\mathbf{G}_{\mathbf{0}}$ and $\mathbf{G}_{\mathbf{1}}$ is negligible.

Game $\mathbf{G}_{\mathbf{2}}$. The game is identical to $\mathbf{G}_{\mathbf{1}}$ except the delegator removes information about $c_{j}$ and $B_{i j}$ from each $Y_{i j}$, i.e., $Y_{i j}=g_{2}^{r_{i j}^{(1)}}$ instead of $g_{2}^{c_{j} B_{i j}+r_{i j}^{(1)}}$. To be able to verify the result of computation, we also update $\mathrm{SK}_{x}$ to compensate for the difference in $Y_{i j}$ 's. We thus add the difference in the $v k_{i}$ 's $\sum_{i=1}^{n_{1}} \sum_{j=1}^{n_{3}} r_{i} c_{j}\left(\sum_{k=1}^{n_{2}} A_{i k} B_{k j}\right)$ to the value of $v k_{1}$ in $\mathbf{G}_{\mathbf{1}}$ while keeping the remaining $v k_{i}$ 's the same as in $\mathbf{G}_{\mathbf{1}}$ (note that ther are other possibilities because only $\sum_{i} v k_{i}$ 's is used). Because the $r_{i j}^{(1)}$ 's are completely random, the distribution of the $Y_{i j}$ 's in $\mathbf{G}_{\mathbf{1}}$ and in $\mathbf{G}_{\mathbf{2}}$ is identical and thus $\operatorname{Pr}\left[T_{2}\right]=\operatorname{Pr}\left[T_{1}\right]$. Now observe that we removed any information about the $c_{j}$ 's from $\mathcal{A}$ 's view.

Let us next analyze $\mathcal{A}$ 's success in winning $\operatorname{Exp}_{\mathcal{A}}^{\operatorname{Ver}}\left(\mathrm{VC}_{m}, f, \kappa\right)$ in $\mathbf{G}_{\mathbf{2}}$. Assuming the $\mathrm{XDH}$ assumption is true, the M-DDH problem is hard in our setting with bilinear maps, i.e., it is hard in $\mathbb{G}_{T}$. Thus, while $\mathcal{A}$ can compute $g_{T}^{r_{i}}$ for each $i,\left(\left\{g_{T}^{r_{i}}\right\}_{1 \leq i \leq n_{1}},\left\{g_{T}^{r_{i} c_{j}}\right\}_{1 \leq i \leq n_{1}, 1 \leq j \leq n_{3}}\right)$ is a partial $\left(n_{1}+n_{3}\right)$-M-DDH tuple and $\mathcal{A}$ can have only a negligible advantage in distinguishing the $g_{T}^{r_{i} c_{j}}$, from random group elements. Now suppose that $\mathcal{A}$ was able to return a tuple $(\hat{C}, \hat{s})$ that differs from correct $(C, s)$, but nevertheless passes verification. Then the returned value satisfies the equation $g_{T} \sum_{i=1}^{n_{1}} \sum_{j=1}^{n_{3}} r_{i} c_{j}\left(C_{i j}-\hat{C}_{i j}\right)=s / \hat{s}$. Because the server has no information about the $c_{j}$ 's and furthermore is unable to distinguish $g_{T}^{r_{i} c_{j}}$, s from random group elements, the only way for $\mathcal{A}$ to create simultaneously valid $C_{i j}-\hat{C}_{i j}$ and $s / \hat{s}$ is to correctly guess the value of $g_{T}^{r_{i} c_{j}}$. The probability of this happening is, however, negligible in $\kappa$ and thus $\operatorname{Pr}\left[T_{2}\right]$ is negligible as well.

Combined with the previous analysis of the differences in the adversarial success between games $\mathbf{G}_{\mathbf{0}}$ and $\mathbf{G}_{\mathbf{2}}$, we obtain that $\mathcal{A}$ 's advantage is negligible in winning the experiment $\mathbf{E x p}_{\mathcal{A}}^{\operatorname{Ver}}\left(\mathrm{VC}_{m}, f, \kappa\right)$ as desired.

\section{Matrix Multiplication in Presence of Lazy Adversary}

Our next construction aims at defending against a rational adversary. Because a rational adversary behaves in the most profitable manner by considering both the compensation paid by the client and the cost endured during the computation, it would be to the adversary's advantage to honestly report all computed results to obtain compensation for the work (rather than report a bogus result that could be detected with overwhelming probability and hence yield a lower reward).

In our solution against rational adversaries $\mathrm{VC}_{r}$, we would like to achieve two features: (i) to force a rational adversary who wishes to maximize its profits to conform to the prescribed protocol and (ii) in case of faulty computation, to pinpoint location of faulty cells by both the server and the client. Realizing both features is achieved by requiring the client to perform only work sublinear in the size of the matrices at the time of computation verification. For the ease of presentation, we describe our solution in a modular manner, where the first scheme support only the first feature and the second presented scheme enhances it with the second feature. 


\subsection{Description of the Base Scheme}

The main idea behind $\mathrm{VC}_{r}$ is similar to that of $\mathrm{VC}_{m}$ : as before, the delegator encodes $A$ into $X$ and $B$ into $Y$ and asks the server to compute products $A \times B$ and $X \times Y$. Similar to $\mathrm{VC}_{m}$, correctness of $A \times B$ is verified by checking a secret relationship between the two matrix products. However, unlike $\mathrm{VC}_{m}$, where the delegator performs the verification itself, in $\mathrm{VC}_{r}$, the delegator further outsources the verification task to the server and only performs one string comparison to confirm correctness of the verification process. The saving in the verification cost comes with slightly increased work during problem generation, but this work is still linear in the size of the input and output. This scheme can be particularly suitable in the setting with three distinct entities (besides the server) such as a doctor who delegates problem generation to lab assistants and patients who verifies the result of the computation returned by the server. The entity performing problem generation (i.e., lab assistant in the above example) is willing to put in additional (one-time) work to benefit routine operations (i.e., verification) by end users (patients).

In our solution, the delegator, as before, produces $X$ as a randomized version of $A$ with its elements of the form $g_{1}^{r_{i} A_{i j}}$ and $Y$ as a randomized version of $B$ with its elements of the form $g_{2}^{c_{j} B_{i j}+w_{j} d_{i}}$. The delegator also releases a new matrix $Z$ formed as $g_{T}^{r_{i} c_{j}}$ to aid the server in producing a proof of correct computation. Unlike $\mathrm{VC}_{m}$, security of which relies on the secrecy of $g_{T}^{r_{i} c_{j}}$, in $\mathrm{VC}_{r}$, we need to incorporate additional secret information in the solution to guarantee security despite public exposure of matrix $Z$. If we treat $Y$ as $\hat{B}+T$, where $\hat{B}=g_{2}^{c_{j} B_{i j}}$ and $T=g_{2}^{w_{j} d_{i}}$, we want to use $X \times T$ as secret information. In order to do that, the cells of $X \times T$ should be indistinguishable from random group elements, and can be produced by the server only if it follows the prescribed protocol. Towards the goal, we incorporate additional randomization into $X$ and $\hat{B}$ by representing their elements as $g_{1}^{r_{i} / m_{j} A_{i j}}$ and $g_{2}^{c_{j} m_{i} B_{i j}}$, respectively, using another random vector $\left\{m_{i}\right\}_{i=1}^{n_{2}}$. The secret verification key is set to be the result of hashing of all cells of $X \times T$. Therefore, to pass verification, the server has to recover all elements of $X \times T$ correctly. Notice that because the server is unable to separate $\hat{B}$ from $T$ in $Y$ and thus compute $X \times T$ by unintended means, the server is forced to compute $X \times Y$ and $A \times B$, determine $X \times \hat{B}$ from $A \times B$ with the help of $Z$, and remove $X \times \hat{B}$ from $X \times Y$ to recover the key. The scheme is given in Figure 2 .

The complexity of ProbGen is $O\left(n_{1} n_{2}+n_{1} n_{3}+n_{2} n_{3}\right)$, i.e., linear in the size of the input and output. The complexity of Compute is dominated by two matrix multiplications resulting in $O\left(n_{1} n_{2} n_{3}\right)$ time. Lastly, the Verify algorithm performs a single string comparison of complexity $O(1)$ and outputs the matrix of size $n_{1} \times n_{3}$.

Security of this solution holds only when each cell $A_{i j}$ of matrix $A$ takes a non-zero value. For that reason, we next describe a mechanism for encoding an arbitrary matrix $M$ into an equivalent matrix $M^{\prime}$ that contains only non-zero values and decoding the result after $M^{\prime}$ is used in the computation.

Matrix encoding: Given $M$ of dimensions $n_{1} \times n_{2}$, choose any value $\ell$ such that $A_{i j}+\ell \neq 0$ for $1 \leq i \leq n_{1}$ and $1 \leq j \leq n_{2}$. To form $M^{\prime}$, add $\ell$ to each element of $M$, i.e., $M_{i j}^{\prime}=M_{i j}+\ell$, and store $\ell$ for future reference.

Matrix decoding: Let $C^{\prime}=M^{\prime} \times N^{\prime}$, where $M^{\prime}\left(N^{\prime}\right)$ is an encoded version of matrix $M$ (resp., $N$ ) using value $\ell_{1}$ (resp., $\ell_{2}$ ) and has dimensions $n_{1} \times n_{2}$ (resp., $n_{2} \times n_{3}$ ). Observe that each element $C_{i j}^{\prime}=\sum_{k=1}^{n_{2}}\left(M_{i k}+\ell_{1}\right)\left(N_{k j}+\ell_{2}\right)$. To recover $C=M \times N$, compute the offset $\Delta_{i j}$ for each element, which equals to $\ell_{2} \sum_{k=1}^{n_{2}} M_{i k}+\ell_{1} \sum_{k=1}^{n_{2}} N_{k j}+n_{2} \ell_{1} \ell_{2}$, and set $C_{i j}=C_{i j}^{\prime}-\Delta_{i j}$. Note that the value $\ell_{2} \sum_{k=1}^{n_{2}} A_{i k}\left(\ell_{1} \sum_{k=1}^{n_{2}} B_{k j}\right)$ is the same for all elements in a single row of matrix $M$ (resp., single column of matrix $N$ ). This means that we only need to compute that value for $n_{1}$ rows of matrix $M$ (resp., $n_{3}$ columns of matrix $N$ ). The overall complexity 
Setup $\left(1^{\kappa}, f\right)$ : Given $f$ that indicates matrix multiplication, using the security parameter $\kappa$ run $\left(p, \mathbb{G}_{1}, \mathbb{G}_{2}, \mathbb{G}_{T}, g_{1}, g_{2}, e\right) \leftarrow \operatorname{Set}\left(1^{\kappa}\right)$ and set Set params $=\left(p, \mathbb{G}_{1}, \mathbb{G}_{2}, \mathbb{G}_{T}, g_{1}, g_{2}, g_{T}=\right.$ $\left.e\left(g_{1}, g_{2}\right), f\right)$. Matrix elements should be representable as values in $\mathbb{Z}_{p}$.

$\operatorname{ProbGen}\left(x=(A, B)\right.$, params): On input two matrices $A$ and $B$ of respective dimensions $n_{1} \times n_{2}$ and $n_{2} \times n_{3}$, perform:

1. Choose $r_{i} \stackrel{R}{\leftarrow} \mathbb{Z}_{p}^{*}$ for $1 \leq i \leq n_{1}, d_{j}, m_{j} \stackrel{R}{\leftarrow} \mathbb{Z}_{p}^{*}$ for $1 \leq j \leq n_{2}$, and $c_{k}, w_{k} \stackrel{R}{\leftarrow} \mathbb{Z}_{p}^{*}$ for $1 \leq k \leq n_{3}$.

2. Compute $X_{i j}=g_{1}^{r_{i} / m_{j} A_{i j}}$ for $1 \leq i \leq n_{1}$ and $1 \leq j \leq n_{2}$.

3. Compute $Y_{i j}=g_{2}^{c_{j} m_{i} B_{i j}+w_{j} d_{i}}$ for $1 \leq i \leq n_{2}$ and $1 \leq j \leq n_{3}$.

4. Compute $Z_{i j}=g_{T}^{c_{j} r_{i}}$ for $1 \leq i \leq n_{1}$ and $1 \leq j \leq n_{3}$.

5. Compute $t_{i}=\sum_{k=1}^{n_{2}} A_{i k} d_{k} / m_{k}$ for $1 \leq i \leq n_{1}$, and $v k_{i j}=t_{i} r_{i} w_{j}$ for $1 \leq i \leq n_{1}$ and $1 \leq j \leq n_{3}$.

6. Compute $s k_{i j}=g_{T}^{v k_{i j}}$ for $1 \leq i \leq n_{1}$ and $1 \leq j \leq n_{3}$, set $s k_{j}=H\left(s k_{1 j}\left\|s k_{2 j}\right\| \ldots \| s k_{n_{1} j}\right)$ for $1 \leq j \leq n_{3}$, and $s k=H\left(s k_{1}\left\|s k_{2}\right\| \ldots \| s k_{n_{3}}\right)$.

7. Set $\mathrm{SK}_{x}=s k, \mathrm{EK}_{x}=($ params, $Z)$, and $\sigma_{x}=(A, B, X, Y)$.

Compute $\left(\mathrm{EK}_{x}=(\right.$ params, $\left.Z), \sigma_{x}=(A, B, X, Y)\right)$ : Execute the following steps:

1. For $j=1, \ldots, n_{3}$ do:

(a) For $1 \leq i \leq n_{1}$, compute

- $V_{i j}^{(1)}=\sum_{k=1}^{n_{2}} A_{i k} B_{k j}$

- $V_{i j}^{(2)}=\prod_{k=1}^{n_{2}} e\left(X_{i k}, Y_{k j}\right)=g_{T}^{\sum_{k=1}^{n_{2}} A_{i k}\left(c_{j} r_{i} B_{k j}+w_{j} d_{k} r_{i} / m_{k}\right)}$

- $\Delta_{i j}=V_{i j}^{(2)} / Z_{i j} V_{i j}^{(1)}$ for $1 \leq j \leq n_{3}$ and $1 \leq i \leq n_{1}$.

2. Compute $s \hat{k}_{j}=H\left(\Delta_{1 j}\left\|\Delta_{2 j}\right\| \ldots \| \Delta_{n_{1} j}\right)$ and $\hat{s k}=H\left(\hat{s k_{1}}\left\|s \hat{k}_{2}\right\| \ldots . \mid \hat{s k}_{n_{3}}\right)$.

3. Set $\sigma_{y}=\left(V^{(1)}, \hat{s k}\right)$.

Verify $\left(\mathrm{SK}_{x}=s k, \sigma_{y}=\left(V^{(1)}, \hat{s k}\right)\right)$ : Verify whether $\hat{s k}=s k$. If the check succeeds, output $V^{(1)}$; otherwise, output $\perp$.

Figure 2: Description of the core scheme $\mathrm{VC}_{r}$ in presence of lazy adversaries.

of computing all offsets is therefore $O\left(n_{1} n_{2}+n_{2} n_{3}\right)$ and the complexity of computing $C$ from $C^{\prime}$ is $O\left(n_{1} n_{3}\right)$.

The decoding computation is simplified when only one of the matrices used in the product was encoded to eliminate zero entries (as in $\mathrm{VC}_{r}$ ). In that case, the offset becomes $\Delta_{i j}=$ $\ell_{1} \sum_{k=1}^{n_{2}} N_{k j}$ assuming that $M$ was the encoded matrix, and the overall complexity of decoding is $O\left(n_{1} n_{3}+n_{2} n_{3}\right)$.

\subsection{Analysis}

To demonstrate correctness of $\mathrm{VC}_{r}$, we show that if the computation was performed correctly, the server is able to recover the key $s k$. That is, an honest server will be able pass verification when $s k$ is honestly computed as $s k=H\left(s k_{1}\left\|s k_{2}\right\| \ldots \| s k_{n_{3}}\right)$ and for each $j=1, \ldots, n_{3}$, we have:

$$
\begin{aligned}
s \hat{k}_{j} & =H\left(\Delta_{1 j}\left\|\Delta_{2 j}\right\| \ldots \| \Delta_{n_{1} j}\right) \\
& =H\left(V_{1 j}^{(2)} / Z_{1 j}{ }^{V_{1 j}^{(1)}}\left\|V_{2 j}^{(2)} / Z_{2 j}{ }^{(1)}\right\| \ldots \| V_{n_{1 j}}^{(2)} / Z_{n_{1 j}}{ }^{V_{n_{1} j}^{(1)}}\right)
\end{aligned}
$$




$$
\begin{aligned}
& =H\left(g_{T}^{\sum_{k=1}^{n_{2}} A_{1 k}\left(w_{j} d_{k} / m_{k}\right) r_{1}}\|\ldots\| g_{T}^{\sum_{k=1}^{n_{2}} A_{n_{1} k}\left(w_{j} d_{k} / m_{k}\right) r_{n_{1}}}\right) \\
& =H\left(\hat{s k}_{1 j}\left\|\hat{s k}_{2 j}\right\| \ldots \| \hat{s k}_{n_{1} j}\right)=s k_{j}
\end{aligned}
$$

That is, since $\hat{s k}_{j}=s k_{j}$ for each $j, \hat{s k}=s k$. Security of our $\mathrm{VC}_{r}$ scheme can be stated as follows:

Theorem 2 If $H$ is a collision-resistant hash function, the $M-D D H$ and XDH assumptions hold, and all elements of $A$ are non-zero, a server that deviates from the correct protocol can only pass verification with a probability negligible in the security parameter.

Proof Similar to the proof of Theorem 1, we proceed with a series of games using the hybrid argument and analyze the adversary's capabilities in the final game.

Game $\mathbf{G}_{\mathbf{0}}$. Define $\mathbf{G}_{\mathbf{0}}$ to be the same as $\operatorname{Exp}_{\mathcal{A}}^{\operatorname{Ver}}\left(\mathrm{VC}_{r}, f, \kappa\right)$.

Game $\mathbf{G}_{\mathbf{1}}$. The game is identical to $\mathbf{G}_{\mathbf{0}}$, except that when generating $Y_{i j}$, the delegator will use random value $r_{i j}^{(1)}$ in $\mathbb{Z}_{p}$ instead of $w_{j} d_{i}$. To be able to verify the result using the original Verify, the delegator also updates each $v k_{i j}$ in $\mathrm{SK}_{x}$ from $r_{i} w_{j} \sum_{k=1}^{n_{2}} A_{i k} d_{k} / m_{k}$ in $\mathbf{G}_{\mathbf{0}}$ to $r_{i} \sum_{k=1}^{n_{2}} A_{i k} r_{k_{j}}^{(1)} / m_{k}$. As before, assuming that $\left(n_{2}+n_{3}\right)-\mathrm{M}-\mathrm{DDH}$ problem is hard, $\left|\operatorname{Pr}\left[T_{1}\right]-\operatorname{Pr}\left[T_{0}\right]\right|$ is negligible.

Game $\mathbf{G}_{\mathbf{2}}$. The game is identical to $\mathbf{G}_{\mathbf{1}}$ except that the delegator removes $c_{j}, m_{i}$, and $B_{i j}$ from each $Y_{i j}$, which is now formed as $g_{2}^{r_{i j}^{(1)}}$ instead of $g_{2}^{c_{j} m_{i} B_{i j}+r_{i j}^{(1)}}$. The delegator also makes corresponding changes to $\mathrm{SK}_{x}$ to ensure that Verify works by setting each $v k_{i j}$ to $\sum_{k=1}^{n_{2}} A_{i k} r_{i}\left(r_{k j}^{(1)} / m_{k}-c_{j} B_{k j}\right)$. Because the $r_{i j}^{(1)}$ 's are completely random, the distribution of the $Y_{i j}$ 's in $\mathbf{G}_{\mathbf{1}}$ and the $Y_{i j}$ 's in $\mathbf{G}_{\mathbf{2}}$ is identical and $\mathcal{A}$ 's view does not change, i.e., $\operatorname{Pr}\left[T_{2}\right]=\operatorname{Pr}\left[T_{1}\right]$.

Game $\mathbf{G}_{3}$. The game is identical to $\mathbf{G}_{\mathbf{2}}$ except that the delegator replaces the $r_{i} / m_{j}$ 's in $X_{i j}$ 's with random values $r_{i j}^{(2)}$ and the $c_{j} r_{i}$ 's used in generating $Z_{i j}$ 's with random values $r_{i j}^{(3)}$. The delegator also sets each $v k_{i j}$ in $\mathrm{SK}_{x}$ to $\sum_{k=1}^{n_{2}} A_{i k}\left(r_{i k}^{(2)} r_{k j}^{(1)}-B_{k j} r_{i j}^{(3)}\right)$ to be able to verify the result of computation. Because anyone with access to the public parameters can compute values equivalent to $X_{i j}$ in $\mathbb{G}_{T}$ (i.e., $g_{T}^{r_{i} A_{i j} / m_{j}}$ ), we treat all values as an instance of a single M-DDH problem. Then assuming the $\left(n_{1}+n_{2}+n_{3}\right)$-M-DDH problem defined on $g_{T}^{r_{i}}$ s $\left(g_{1}^{r_{i}}, \mathrm{~s}\right), g_{T}^{c_{j}}$ s, and $g_{T}^{1 / m_{j}}, \mathrm{~s}\left(g_{1}^{1 / m_{j}}\right.$, s $)$ is hard, the difference in $\mathcal{A}$ 's view between $\mathbf{G}_{\mathbf{2}}$ and $\mathbf{G}_{\mathbf{3}}$ is negligible.

Game $\mathbf{G}_{\mathbf{4}}$. The game is the same as $\mathbf{G}_{\mathbf{3}}$ except that each $X_{i j}=g_{1}^{r_{i j}^{(2)}}$ instead of $g_{1}^{r_{i j}^{(2)}} A_{i j}$. Additionally, each $v k_{i j}$ in $\mathrm{SK}_{x}$ is set to $\sum_{k=1}^{n_{2}}\left(r_{i k}^{(2)} r_{k j}^{(1)}-A_{i k} B_{k j} r_{i j}^{(3)}\right)$. Assuming each $A_{i j}$ is non-zero, $\mathcal{A}$ 's view is unchanged from $\mathbf{G}_{\mathbf{3}}$.

Now observe that we completely removed any information used in constructing key $s k$ from $\mathcal{A}$ 's view. This implies that $\mathcal{A}$ is unable to pass verification with more than negligible probability by guessing or performing unrelated computation. Next, we show that the only way to pass verification with non-negligible probability is to faithfully follow the protocol and compute correct key $s k$. Specifically, to match $s k$ computed as $H\left(s k_{1}\left\|s k_{2}\right\| \ldots \| s k_{n_{3}}\right), \mathcal{A}$ needs to produce correct $s k_{i}$ for $i=1, \ldots, n_{3}$ because of the collision-resistance of hash function $H$. Similarly, because each $s k_{i}$ is formed as $H\left(\Delta_{1 j}\left\|\Delta_{2 j}\right\| \ldots \| \Delta_{n_{1} j}\right), \mathcal{A}$ has to have $\Delta_{i j}$ 's to produce correct $s k_{i}$ and pass verification. Furthermore, observe that $\Delta_{i j}$ is a function of $d_{i}, w_{j}$, and $1 / m_{k}$ (more precisely, a function of their product) and information about $d_{i} w_{j}$ is encoded in $Y$ and information about $1 / m_{k}$ is encoded in $X$ (and no other values contain information about $d_{i}, w_{j}$, or $1 / m_{k}$ ). Then the only way for $\mathcal{A}$ to derive $d_{i} w_{j} / m_{k}$ is to use $X$ and $Y$ as a whole and apply the pairing operation which will result in the desired product. Now notice that by doing this, $\mathcal{A}$ will introduce the term $g_{T} c_{j} r_{i} \sum_{k=1}^{n_{2}} A_{i k} B_{k j}$ that protects $\Delta_{i j}$. To remove it, $\mathcal{A}$ has to use $Z_{i j}$, which is the only value that is a function of $c_{j}$. 
Thus, $\mathcal{A}$ is forced to compute $Z_{i j}{ }^{(1)}$ to obtain correct $\Delta_{i j}$, which necessitates faithful computation of $V^{(1)}$ that corresponds to $A \times B$. By executing the above steps, $\mathcal{A}$ is forced to follow the protocol, which is the only way to produce correct $s k$ with a non-negligible probability.

Assumption 2 If the server returns $\sigma_{y}=\left(V^{(1}, \hat{s k}\right)$ such that $\hat{s k}=s k$, but $V^{(1)} \neq C$, there is a non-negligible probability that the error will be detected by the client.

This assumption is crucial to our result and can be realized by outsourcing a small (but nonnegligible) fraction $0<\rho<1$ of all tasks to a second independent server. For example, for each task the client chooses random $v \in[0,1]$, and if $v \leq \rho$, the client uses two (non-colluding) servers for independently outsourcing the task and compares the returned products $C$ afterwards. If the servers are non-colluding and at least one returned $C$ is incorrect, the client will be able to detect misbevior with non-negligible probability. This implies that if the server performed the work to pass the verification test, it will be incentivized to return correct $C$.

Theorem 3 If Assumptions 1 and 2 and assumptions of Theorem 2 hold, $\mathrm{VC}_{r}$ is secure in presence of rational adversaries according to Definition 6.

Proof of Theorem 3 Based on Assumptions 1 and 2 and Theorem 2, we show $\mathrm{VC}_{r}$ satisfies the three conditions specified in Definition 6, and hence is a rational argument system.

It is obvious that if the server follows the prescribed protocol in a faithful manner, he is able to return a correct answer with probability 1. Furthermore, Theorem 2 states that, if the server deviates from the prescribed protocol, he only has a negligible probability to pass the verification test. Let $\mu(|x|)$ denote such a probability, where $x$ is the client's input. Also, let $R(|x|)$ and $C(|x|)$ denote the server's reward and cost, respectively, which according to Assumption 1 are polynomial in $|x|$. Therefore, if the reward is 0 when the server is caught cheating, the expected reward for an unfaithful prover that did not perform the computation is

$$
E[\operatorname{reward}((\hat{P}, V)(x))]=(1-\mu(|x|)) \cdot 0+\mu(|x|) R(|x|) .
$$

If, however, the server performed the computation correctly, but did not return correct matrix $C$, its expected reward is $p_{1} \cdot(-C(|x|))+\left(1-p_{1}\right)\left(R(|x|-C(|x|))\right.$, where $p_{1}$ denotes the probability that the problem is detected at a later point, in which case the compensation is 0 . Let $p_{2}$ denote the probability that a cheating server follows the first strategy (i.e., does not perform the computation) and $\left(1-p_{2}\right)$ will be the probability that it follows the second strategy (i.e., performs the computation, but returns incorrect matrix $C$ ). This gives us the overall expected reward

$$
E[\operatorname{reward}((\hat{P}, V)(x))]=p_{2} \mu(|x|) R(|x|)+\left(1-p_{2}\right)\left(\left(1-p_{1}\right)(R(|x|)-C(|x|))-p_{1} C(|x|)\right)
$$

In the case of truthful behavior, $E[\operatorname{reward}((P, V)(x))]=R(|x|)-C(|x|)>0$. Because in the first cheating strategy the expected reward $(\mu(|x|) R(|x|))$ is negligible and in the second cheating strategy the reward is always less than the reward of honest behavior, we obtain that

$$
E[\operatorname{reward}((P, V)(x))]>E[\operatorname{reward}((\hat{P}, V)(x))] .
$$

Regarding the third property, if $\operatorname{Pr}[\operatorname{output}((\hat{P}, V)(x)) \neq f(x)] \geq p(|x|)^{-1}=p$, and if we use $E\left[\right.$ reward $\left._{\hat{P}}\right]$ to denote the overall reward of a dishonest server that is computed in the proof of second property, we have

$$
E[\operatorname{reward}((\hat{P}, V)(x))]=p \cdot E\left[\operatorname{reward}_{\hat{P}}\right]+(1-p) E[\operatorname{reward}((P, V)(x))]
$$


Therefore, $E[\operatorname{reward}((P, V)(x))]-E[\operatorname{reward}((\hat{P}, V)(x))]=p\left(\left(p_{1}+p_{2}-p_{1} p_{2}\right)(R(|x|)-C(|x|))+\right.$ $\left.p_{1}\left(1-p_{2}\right) C(|x|)-p_{2} \mu(|x|) R(|x|)\right)$. Because $p$ and $p_{1}$ are positive non-negligible probabilities, $\left(p_{1}+p_{2}-p_{1} p_{2}\right) \geq p_{1}, p_{1}\left(1-p_{2}\right) \geq 0$, and $p_{2} \mu(|x|) R(|x|)$ is a negligible value, we can represent the above difference in expected rewards using another non-negligible positive polynomial $q(|x|)^{-1}$, and have the third property satisfied.

The above means that following the prescribed protocol and producing correct matrix products $A \times B$ and $X \times Y$ is the most profitable strategy for a rational adversary.

\subsection{Description of Enhanced Scheme}

In this section, we propose an enhanced scheme that supports chaining and allows honest parties to pinpoint faulty cells in case of computation corruption or intentional deviation from the prescribed computation using a single mechanism. That is, recall that this feature makes it difficult for a dishonest server to continue with the next stage of the computation if it was not carried out correctly at the current stage and allows the client to efficiently identify the first stage at which a fault occurred. It also allows the cloud itself to detect a problem with the computation (in case of compromise or malware infection). The basic idea behind the solution is that the client divides the entire computation into $n_{3}$ sub-computations. The keys $s k_{i}$ are formed as before, but now the $i$ th key is used to encode the inputs of the $(i+1)$ th sub-computation. The server is able to recover the $(i+1)$ th sub-key only if it executes sub-computations at stages 1 through $i$ correctly. Upon computation completion, the verifier receives the last key from the server and examines its correctness. If the verifier notices a discrepancy between the returned key and its expected value, he will ask the server to return all the keys generated throughout the computation. The verifier then applies a procedure similar to binary search to locate the first incorrect key, which corresponds to the first sub-computation that has been executed incorrectly. This operation can be implemented in $O(\log n)$ steps, where $n$ is the total number of sub-computations. The server will also be able to examine the correctness of the first $i$ sub-computations that have been executed so far by verifying that the inputs of $(i+1)$ th sub-computation could be decoded correctly using $s k_{i}$. If this check fails, this serves as a notification to the server of the existence of faulty cells and the server can suspend the computation to identify faulty sells among the cells computed so far.

The detail of our solution can be described as follows: The client generates five matrices $\{A, B, X, Y, Z\}$ and forms keys $s k_{i}$ as in the base scheme. Now the computation of the $i$ th column of matrices $A \times B$ and $X \times Y$ is considered to be the $i$ th sub-computation. The client then blinds each element of the $(i+1)$ th column of matrices $B$ and $Y$ by XORing them with a pseudo-random string. This pseudo-random string is produced using $s k_{i}$ as the secret key to a pseudo-random function PRF, which is evaluated on the cell's row and column indices together with a unique identifier for each matrix to guarantee uniqueness of the input/output. In order to remove blinding and recover the next sub-computation, the server needs to produce correct $s k_{i}$ as before (by computing the $i$ th column of two matrix products) and evaluate the PRF on that key to reproduce the random mask. This will allow the server to recover the inputs for the $(i+1)$ th column of matrix $B$ and $Y$, i.e., the $(i+1)$ th sub-computation, and continue the computation. We make the size of the pseudo-random string output by PRF to be longer than the size of the matrix elements they mask so that the remaining bits can be used to verify that the input to the $(i+1)$ th sub-computation was decoded correctly. That is, we append a zero string of a predefined size to each input before encoding, and the server can use it to verify that decoding was successful (and if it was not, investigate the reason for the failure). At the end of the computation, the server recovers and returns the last key $s k_{n_{3}}$, which the client compares to the expected key and accepts the result of the computation if the verification succeeds. The scheme is given in Figure 3. 
$\operatorname{Setup}\left(1^{\kappa}, f\right)$ : The same as in $\mathrm{VC}_{r}$.

$\operatorname{ProbGen}(x=(A, B)$, params): Given matrices $A$ and $B$, perform:

1. Compute $X, Y, Z$, and $\left\{s k_{i}\right\}_{i=1}^{n_{3}}$ as in $\mathrm{VC}_{r}$.

2. Set $\hat{B}_{i 1}=B_{i 1}$ and $\hat{Y}_{i 1}=Y_{i 1}$ for $1 \leq i \leq n_{2}$; and also set

- $\hat{B}_{i j}=\operatorname{PRF}_{s k_{j-1}}(i\|j\| 0) \oplus\left(B_{i j} \| 0^{\lambda}\right)$

- $\hat{Y}_{i j}=\operatorname{PRF}_{s k_{j-1}}(i\|j\| 1) \oplus\left(Y_{i j} \| 0^{\lambda}\right)$

for $1 \leq i \leq n_{2}$ and $2 \leq j \leq n_{3}$, where $\lambda$ is a correctness parameter.

3. Set $\mathrm{SK}_{x}=\left\{s k_{i}\right\}_{i=1}^{n_{3}}, \overline{\mathrm{EK}}_{x}=($ params, $Z)$, and $\sigma_{x}=(A, \hat{B}, X, \hat{Y})$.

Compute $\left(\mathrm{EK}_{x}=(\right.$ params,$\left.Z), \sigma_{x}=(A, \hat{B}, X, \hat{Y})\right)$ : Execute steps:

1. Set $U_{i 1}^{(1)}=\hat{B}_{i 1}$ and $U_{i 1}^{(2)}=\hat{Y}_{i 1}$ for $1 \leq i \leq n_{2}$.

2. For $j=1, \ldots, n_{3}$ do:

(a) For $1 \leq i \leq n_{1}$, compute

- $V_{i j}^{(1)}=\sum_{k=1}^{n_{2}} A_{i k} U_{k j}^{(1)}$

- $V_{i j}^{(2)}=\prod_{k=1}^{n_{2}} e\left(X_{i k}, U_{k j}^{(2)}\right)$

(b) Let $\Delta_{i j}=V_{i j}^{(2)} / Z_{i j} V_{i j}^{(1)}$ for $1 \leq i \leq n_{1}$. Compute $s \hat{k}_{j}=H\left(\Delta_{1 j}\left\|\Delta_{2 j}\right\| \ldots \| \Delta_{n_{1} j}\right)$.

(c) If $j \neq n_{3}$, for $1 \leq i \leq n_{2}$ compute

- $U_{i j+1}^{(1)} \| W_{1}=\hat{B}_{i j+1} \oplus \operatorname{PRF}_{s \hat{k}_{j}}(i\|j+1\| 0)$

- $U_{i j+1}^{(2)} \| W_{2}=\hat{Y}_{i j+1} \oplus \operatorname{PRF}_{s \hat{k}_{j}}(i\|j+1\| 1)$

If $W_{1}$ or $W_{2}$ is not equal to $0^{\lambda}$, report an error and abort.

3. Set $\sigma_{y}=\left(V^{(1)}, \hat{s k}_{n_{3}}\right)$.

$\operatorname{Verify}\left(\mathrm{SK}_{x}=\left\{s k_{i}\right\}_{i=1}^{n_{3}}, \sigma_{y}=\left(V^{(1)}, s k_{n_{3}}\right)\right)$ : Verify whether $s \hat{k}_{n_{3}}=s k_{n_{3}}$. If the check succeeds, output $V^{(1)}$. Otherwise, retrieve all $\left\{\hat{s k}_{i}\right\}_{i=1}^{n_{3}}$ from the server and find the smallest index $i$ such that $\hat{k}_{i} \neq s k_{i}$ using binary search.

Figure 3: Description of scheme $\mathrm{VC}_{r}$ that incorporates chaining and allows for fast location of an error in the computation.

The complexity of ProbGen and Compute algorithms remain the same as the basic scheme, i.e., $O\left(n_{1} n_{2}+n_{1} n_{3}+n_{2} n_{3}\right)$ and $O\left(n_{1} n_{2} n_{3}\right)$, respectively. The Verify algorithm performs a single string comparison of complexity $O(1)$ and outputs a matrix of size $n_{1} \times n_{3}$ in case of no errors. If the check, however, fails, the client retrieves $n_{3}$ keys and additionally performs $O\left(\log n_{3}\right)$ work.

\subsection{Analysis}

To show correctness, it is trivial to demonstrate that the server is able to recover the inputs for the next sub-computation upon computing key $s k_{j}$ for each $j$. Because the server can produce each random mask by computing $\operatorname{PRF}_{s k_{j-1}}(i\|j\| i d)$, it is able to obtain the inputs and complete the computation.

We start showing security of the enhanced $\mathrm{VC}_{r}$ scheme with the following claim:

Lemma 1 If $H$ is a collision-resistant hash function, PRF is a pseudo-random function, the $M$ $D D H$ and $X D H$ assumptions hold, and all elements of $A$ are non-zero, it is computationally difficult 
for the server who did not follow the prescribed computation for stages 1 through $i$ to recover the input for $(i+1)$ th sub-computation for $i \geq 1$.

Proof We prove this by induction on $i$.

Basic step: Let $i=1$. The server receives inputs to the first sub-computation in the clear and would like to recover the inputs for the next sub-computation. First, notice that the inputs to the second-computation are protected by the output of PRF evaluated on unique points. This means that a computationally-bounded server will be unable to learn any information about the inputs with greater than negligible probability without access to $s k_{1}$. To recover $s k_{1}$, however, the only option for the server is to comply with the prescribed computation, using exactly the same argument as in the proof of Theorem 2.

We obtain that an adversary who deviates from the prescribed computation is unable to recover $s k_{1}$ and thus the inputs for the second sub-computation, as claimed.

Induction step: Suppose that the claim is true for some $k=i$ and we show that it is true for $k=i+1$. The argument proceeds similarly to the basic step above. That is, by induction we know that it is infeasible to recover inputs for the $i$ th sub-computation without following the prescribed computation. Therefore, if the server was able to recover the inputs to the $i$ th sub-computation, it must be difficult to recover the inputs to the $(i+1)$ th sub-computation without honestly carrying out the computation for the $i$ th sub-computation. The security argument proceeds in the same way as in the basic step.

If the server was not able to recover the inputs to the $i$ th sub-computation, it is still infeasible to recover the inputs to the $(i+1)$ th sub-computation due to the security of the PRF function used. This completes the proof.

Theorem 4 If $H$ is a collision-resistant hash function, PRF is a pseudo-random function, the $M$ $D D H$ and $X D H$ assumptions hold, and all elements of $A$ are non-zero, the server that deviates from the correct protocol can only pass verification with a probability negligible in the security parameter.

Proof According to Lemma 1, if the server deviates at any stage of the protocol except for the last one, it will be computationally difficult for him to recover the input of the last stage, which leads to a negligible probability to pass verification. Moreover, if the server deviates at the last stage, according to Theorem 2, it also results in a negligible success probability. This means if the server deviates from the prescribed protocol, he can only pass verification with a probability negligible in the security parameter.

Theorem 5 If Assumptions 1 and 2 and assumptions of Theorem 4 hold, $\mathrm{VC}_{r}$ that incorporates chaining is secure in presence of rational adversaries according to Definition 6.

The proof is similar to that of Theorem 3 .

Corollary 1 If the result of the returned computation does not verify, the client will be able to identify the first faulty sub-computation using $O\left(\log \left(n_{3}\right)\right)$ string comparisons.

Proof Based on Lemma 1, if a key $s k_{i}$ was not computed correctly, it is computationally difficult to recover all consecutive keys $s k_{i+1}, \ldots, s k_{n_{3}}$. This means that to identify the first faulty sub-task, the client needs to find $i$ such that the server's $s k_{i} \neq s k_{i}$, but $s k_{i-1}=s k_{i-1}$. Finding such an $i$ using binary search can be accomplished in $O\left(\log \left(n_{3}\right)\right)$ steps. 


\section{Support for Matrix Privacy}

In this section, we extend both of our previous constructions, $\mathrm{VC}_{m}$ and $\mathrm{VC}_{r}$, to incorporate privacy (or data secrecy) protection of input/output matrices. We denote the resulting schemes as $\mathrm{VC}_{m}^{p}$ and $\mathrm{VC}_{r}^{p}$, respectively. In more detail, we would like to ensure that the server who executes the outsourced task is unable to learn any information regarding input matrices $A$ and $B$, and consecutively their product $A \times B$, throughout the computation. We thus modify both schemes to achieve this goal, while still preserving their original properties of verifiable computation.

\subsection{Support for Matrix Privacy in Malicious Setting}

In $\mathrm{VC}_{m}^{p}$, we wish to guarantee that the server is unable to infer any information about the input matrices $A$ and $B$ from $\sigma_{x}$ and $\mathrm{EK}_{x}$ that ProbGen outputs. Because $\mathrm{EK}_{x}$ consists of only public parameters, we need to concentrate on $\sigma_{x}$ that consists of matrices $A, B, X$, and $Y$. To protect $A$ and $B$, our solution encodes them using a homomorphic encryption scheme (e.g., BGN encryption [15]) that supports one multiplication and an unlimited number of additions on messages in encrypted form. This allows for matrix multiplication $A \times B$ to be privately carried out on encrypted data. With respect to the remaining matrices, notice that no changes to $Y$ are necessary. That is, the term $g_{2}^{w_{j} d_{i}}$ protects each element of matrix $B$ that matrix $Y$ encodes, assuming that the M-DDH assumption holds. Matrix $X$ in its original form, however, can disclose information about the elements of matrix $A$. To prevent this disclosure, we encode its elements in a form similar to that of matrix $Y$, by incorporating a random term $g_{1}^{h_{j} u_{i}}$ into each element of $Y$. The detailed protocol is presented in Figure 4.

To protect the original elements of matrix $X$, the delegator chooses additional random values $u_{i}$ and $h_{j}$ in ProbGen, which are incorporated into the elements of $X$. Because the format of the elements in $X$ is changed from $g_{1}^{r_{i} A_{i j}}$ to $g_{1}^{r_{i} A_{i j}+h_{j} u_{i}}$, the key $v k$ should also be updated accordingly to satisfy the verification equation. This change does not affect the complexity of ProbGen, which remains as $O\left(n_{1} n_{2}+n_{2} n_{3}\right)$. In Compute, the server executes the protocol in the same way as in $\mathrm{VC}_{m}$ except that the server now performs matrix multiplication on ciphertexts $\operatorname{Enc}\left(A_{i j}\right)$ and $\operatorname{Enc}\left(B_{i j}\right)$ rather than cleartext and returns the encrypted matrix $\operatorname{Enc}(C)$ to the client. The complexity of Compute is still dominated by two matrix multiplications resulting in $O\left(n_{1} n_{2} n_{3}\right)$ time. In Verify, the client decrypts $\operatorname{Enc}(C)$ to obtain $C$ in the clear and then follows the verification procedure of $\mathrm{VC}_{m}$. As before, the procedure has complexity $O\left(n_{1} n_{3}\right)$ (i.e., linear in the size of the output). Output has the same complexity $O\left(n_{1} n_{3}\right)$.

\subsubsection{Analysis}

To demonstrate correctness, we show that if the computation was performed correctly, Verify output 1, the delegator recovers product $A \times B$. We have:

$$
\begin{aligned}
g_{T}^{\sum_{i=1}^{n_{1}} r_{i}\left(\sum_{j=1}^{n_{3}} c_{j} C_{i j}\right)+v k_{i}} & =\prod_{i=1}^{n_{1}} g_{T}^{r_{i} \sum_{j=1}^{n_{3}} c_{j} C_{i j}+v k_{i}}=\prod_{i=1}^{n_{1}} \prod_{j=1}^{n_{3}} g_{T}^{r_{i} c_{j} C_{i j}} \times \prod_{i=1}^{n_{1}} g_{T}^{v k_{i}} \\
& =\prod_{i=1}^{n_{1}} \prod_{j=1}^{n_{3}} g_{T}^{\sum_{k=1}^{n_{2}} r_{i} c_{j} A_{i k} B_{k j}} \times \prod_{i=1}^{n_{1}} \prod_{j=1}^{n_{3}} g_{T}^{\sum_{k=1}^{n_{2}}\left(A_{i k} d_{k} r_{i} w_{j}+h_{k} B_{k j} u_{i} c_{j}+h_{k} d_{k} w_{j} u_{i}\right)} \\
& =\prod_{i=1}^{n_{1}} \prod_{j=1}^{n_{3}} \prod_{k=1}^{n_{2}} e\left(g_{1}^{r_{i} A_{i k}+u_{i} h_{k}}, g_{2}^{c_{j} B_{k j}+w_{j} d_{k}}\right)=\prod_{i=1}^{n_{1}} \prod_{j=1}^{n_{3}} \prod_{k=1}^{n_{2}} e\left(X_{i k}, Y_{k j}\right)=s
\end{aligned}
$$

The data protection property of $\mathrm{VC}_{m}^{p}$ construction can be stated as follows: 
$\operatorname{Setup}\left(1^{\kappa}, f\right)$ : The same as in $\mathrm{VC}_{m}$.

$\operatorname{ProbGen}\left(x=(A, B)\right.$, params): On input two matrices $A$ and $B$ of respective dimensions $n_{1} \times n_{2}$ and $n_{2} \times n_{3}$, perform:

1. Choose $r_{i}, u_{i} \stackrel{R}{\leftarrow} \mathbb{Z}_{p}^{*}$ for $1 \leq i \leq n_{1}, d_{j}, h_{j} \stackrel{R}{\leftarrow} \mathbb{Z}_{p}^{*}$ for $1 \leq j \leq n_{2}$, and $c_{k}, w_{k} \stackrel{R}{\leftarrow} \mathbb{Z}_{p}^{*}$ for $1 \leq k \leq n_{3}$.

2. Compute $w=\sum_{j=1}^{n_{3}} w_{j}$ and then $t_{i}=\sum_{k=1}^{n_{2}} A_{i k} d_{k}$ and $v k_{i}=r_{i} t_{i} w$ for $1 \leq i \leq n_{1}$.

3. Compute $m_{i}=\sum_{k=1}^{n_{2}} h_{k} B_{k i}$ for $1 \leq i \leq n_{3}, c=\sum_{i=1}^{n_{3}} c_{i} m_{i}$, and $v k_{i}=v k_{i}+u_{i} c$ for $1 \leq i \leq n_{1}$.

4. Compute $h=\sum_{k=1}^{n_{2}} h_{k} d_{k}$ and $v k_{i}=v k_{i}+u_{i} h w$ for $1 \leq i \leq n_{1}$.

5. Compute $X_{i j}=g_{1}^{r_{i} A_{i j}+h_{j} u_{i}}$ for $1 \leq i \leq n_{1}$ and $1 \leq j \leq n_{2}$.

6. Compute $Y_{i j}=g_{2}^{c_{j} B_{i j}+w_{j} d_{i}}$ for $1 \leq i \leq n_{2}$ and $1 \leq j \leq n_{3}$.

7. Setup homomorphic encryption scheme Enc; denote by params ${ }_{E}$ the scheme's public parameters and by $d k$ private decryption key.

8. Encrypt each element of $A$ and $B$ to produce $\operatorname{Enc}\left(A_{i k}\right)$ and $\operatorname{Enc}\left(B_{k j}\right)$ for $1 \leq i \leq n_{1}$, $1 \leq k \leq n_{2}$, and $1 \leq j \leq n_{3}$.

9. Set $\mathrm{SK}_{x}=\left(\left\{c_{i}\right\}_{i=1}^{n_{3}},\left\{v k_{i}\right\}_{i=1}^{n_{1}},\left\{r_{i}\right\}_{i=1}^{n_{1}}, d k\right), \mathrm{EK}_{x}=$ (params, params $E$ ), and $\sigma_{x}=$ $(\operatorname{Enc}(A), \operatorname{Enc}(B), X, Y)$.

Compute $\left(\operatorname{EK}_{x}, \sigma_{x}=(\operatorname{Enc}(A), \operatorname{Enc}(B), X, Y),\right)$ : Given $\sigma_{x}$, execute:

1. Compute $\operatorname{Enc}(C)=\operatorname{Enc}(A) \times \operatorname{Enc}(B)$.

2. Compute $s=\prod_{i=1}^{n_{1}} \prod_{j=1}^{n_{3}} \prod_{k=1}^{n_{2}} e\left(X_{i k}, Y_{k j}\right)$.

3. Set $\sigma_{y}=(\operatorname{Enc}(C), s)$.

$\operatorname{Verify}\left(\mathrm{SK}_{x}=\left(\left\{c_{i}\right\}_{i=1}^{n_{3}},\left\{v k_{i}\right\}_{i=1}^{n_{1}},\left\{r_{i}\right\}_{i=1}^{n_{1}}, d k\right), \sigma_{y}=(\operatorname{Enc}(C), s)\right)$ : Decrypt $\operatorname{Enc}(C)$ using $d k$ to recover $C$ and verify whether $g_{T}^{\sum_{i=1}^{n_{1}} r_{i}\left(\sum_{j=1}^{n_{3}} c_{j} C_{i j}\right)+v k_{i}}=s$. If the check succeeds, output 1 ; otherwise, output 0 .

Output $\left(\mathrm{SK}_{x}=\left(\left\{c_{i}\right\}_{i=1}^{n_{3}},\left\{v k_{i}\right\}_{i=1}^{n_{1}},\left\{r_{i}\right\}_{i=1}^{n_{1}}, d k\right), \sigma_{y}=(\operatorname{Enc}(C), s)\right)$ : Decrypt $\operatorname{Enc}(C)$ using $d k$ to recover and output $C$ or $\perp$ in case of failure.

Figure 4: Description of scheme $\mathrm{VC}_{m}^{p}$ for malicious adversaries that achieves data privacy.

Theorem 6 Assuming that the $M-D D H$ and $X D H$ problems are hard and Enc is a semantically secure encryption scheme, the verifiable computation scheme $\mathrm{VC}_{m}^{p}$ achieves data secrecy according to Definition 7.

Proof First, notice that the input matrices $A$ and $B$ are protected with secure encryption. This means that we only need to concentrate on the remaining matrices $X$ and $Y$ that the server receives. The goal is to ensure that no information about the inputs is revealed from $X$ or $Y$ throughout the computation. Towards the goal, we proceed with a series of games using the hybrid argument and analyze the adversary's capabilities in the final game.

Game $\mathbf{G}_{\mathbf{0}}$. Define $\mathbf{G}_{\mathbf{0}}$ to be the same as $\operatorname{Exp}_{\mathcal{A}}^{\text {Priv }}\left(\mathrm{VC}_{m}^{p}, f, \kappa\right)$.

Game $\mathbf{G}_{\mathbf{1}}$. The game is identical to game $\mathbf{G}_{\mathbf{0}}$, except that when generating $X_{i j}$ and $Y_{i j}$, the delegator will use random values $r_{i j}^{(1)}$ and $r_{i j}^{(2)}$ from $\mathbb{Z}_{p}$ instead of $h_{j} u_{i}$ and $w_{j} d_{i}$, respectively. To be able to verify the result using the original Verify, the delegator correspondingly updates the $v k_{i}$ 's in $\mathrm{SK}_{x}$. That is, the delegator computes $v k_{i j}=r_{i} \sum_{k=1}^{n_{2}} \sum_{j=1}^{n_{3}} A_{i k} r_{k j}^{(2)}+c_{j} \sum_{i=1}^{n_{1}} \sum_{k=1}^{n_{2}} r_{i k}^{(1)} B_{k j}+$ 
$\sum_{k=1}^{n_{2}} r_{i k}^{(1)} r_{k j}^{(2)}$ for each $1 \leq i \leq n_{1}$ and $1 \leq j \leq n_{3}$ and then sets $v k_{i}=\sum_{j=1}^{n_{3}} v k_{i j}$ for $1 \leq i \leq n_{1}$. As before, assuming that $\left(n_{1}+n_{2}\right)-\mathrm{M}-\mathrm{DDH}$ and $\left(n_{2}+n_{3}\right)-\mathrm{M}-\mathrm{DDH}$ problems are hard, the difference in the adversary's view between $\mathbf{G}_{\mathbf{1}}$ and $\mathbf{G}_{\mathbf{0}}$ is negligible.

Game $\mathbf{G}_{\mathbf{2}}$. The game is identical to $\mathbf{G}_{\mathbf{1}}$ except that the delegator removes information about $r_{i}$ and $A_{i j}$ from each $X_{i j}$, which is now formed as $g_{1}^{r_{i j}^{(1)}}$ instead of $g_{1}^{r_{i} A_{i j}+r_{i j}^{(1)}}$. Similarly, the delegator removes information about $c_{j}$ and $B_{i j}$ from each $Y_{i j}$, which is now formed as $g_{2}^{r_{i j}^{(2)}}$ instead of $g_{2}^{c_{j} B_{i j}+r_{i j}^{(2)}}$. To ensure consistency, the delegator also updates $v k_{i}$ 's in $\mathrm{SK}_{x}$ to $v k_{i}=$ $\sum_{j=1}^{n_{3}} \sum_{k=1}^{n_{2}}\left(r_{i k}^{(1)} r_{k j}^{(2)}-r_{i} c_{j} A_{i k} B_{k j}\right)$. Because the $r_{i j}^{(1)}$ 's $\left(r_{i j}^{(2)}\right.$ 's) are completely random, the distributions of $X_{i j}$ 's (resp., $Y_{i j}$ 's) in $\mathbf{G}_{\mathbf{1}}$ and the $X_{i j}$ 's (resp., $Y_{i j}$ 's) in $\mathbf{G}_{\mathbf{2}}$ are identical and the adversary's view does not change.

Now observe that we completely eliminated any information about the inputs $A$ and $B$ from the adversary's view, which makes the probability of the server winning game $\mathbf{G}_{\mathbf{2}}$ exactly $1 / 2$. Combined with the previous analysis of differences in the adversarial success between games $\mathbf{G}_{\mathbf{0}}$ and $\mathbf{G}_{\mathbf{2}}$, we obtain the adversary's advantage is negligible in winning the experiment $\mathbf{E x p}_{\mathcal{A}}^{\text {Priv }}\left(\mathrm{VC}_{m}^{p}, f, \kappa\right)$.

Theorem 7 Assuming that the $M-D D H$ and XDH problems are hard, the verifiable computation scheme $\mathrm{VC}_{m}^{p}$ is secure according to Definition 5.

Proof There are two differences in the adversary's view between $\mathrm{VC}_{m}$ and $\mathrm{VC}_{m}^{p}$ : (i) in $\mathrm{VC}_{m}^{p}$, the input matrices $A$ and $B$ are sent to the server encrypted and (ii) in $\mathrm{VC}_{m}^{p}$, each element of matrix $X$ includes an additional random term $g_{1}^{h_{j} u_{i}}$. These differences, however, do not give any advantage to the adversary in winning the security game $\operatorname{Exp}_{\mathcal{A}}^{\operatorname{Ver}}\left(\mathrm{VC}_{m}^{p}, f, \kappa\right)$. Therefore, the proof proceeds similar to the proof of Theorem 1 and the claim still holds.

\subsection{Support for Matrix Privacy in Lazy Setting}

Now we would like to modify $\mathrm{VC}_{r}$ to add data protection. Unlike $\mathrm{VC}_{m}$, where the computation of product matrix $C$ and verification value $s$ can be carried out independently, in $\mathrm{VC}_{r}$ the server needs to perform these two computations together in order to produce correct key $\hat{s k}$ used for verification purposes. A direct consequence of the difference is that we can no longer apply arbitrary encryption algorithm to matrices $A$ and $B$ because randomness used in ciphertexts will lead to the delegator's inability to properly compute $\hat{s k}$. To resolve the issue, we encode $A$ and $B$ using a similar mechanism to that of forming matrices $X$ and $Y$. That is, we use the product of two newly generated random values (such as in the form of $w_{j} d_{i}$ in $Y_{i j}$ ) to protect each individual element in $A$ and $B$, and correspondingly update $v k_{i j}$ to allow for correct verification. Description of how to modify input matrices to contain only non-zero values was given in Section 5.1. Our $\mathrm{VC}_{r}^{p}$ construction is given in Figure 5.

The differences from $\mathrm{VC}_{r}$ is that in ProbGen the client now generates additional random values and uses them in combination with other values to form new matrices $X^{\prime}$ and $Y^{\prime}$ from input matrices $A$ and $B$ in a similar way $X$ and $Y$ are formed. Furthermore, there is now no need for the client to produce matrix $Z$ as part of the evaluation key because secret random values $c_{j}$ 's and $r_{i}$ 's are now incorporated into $X^{\prime}$ and $Y^{\prime}$. The overall complexity of ProbGen is still $O\left(n_{1} n_{2}+n_{1} n_{3}+n_{2} n_{3}\right)$. In Compute, the server performs almost the same computation as in $\mathrm{VC}_{r}$, except that in order to produce $V^{(1)}$, the server needs to perform $n_{1} n_{2} n_{3}$ pairing operations instead of modular exponentiations. The overall complexity of Compute is still $O\left(n_{1} n_{2} n_{3}\right)$. In Verify, the verification procedure is a single comparison and therefore has complexity $O(1)$. If the verification 
$\operatorname{Setup}\left(1^{\kappa}, f\right)$ : The same as in $\mathrm{VC}_{r}$.

$\operatorname{ProbGen}\left(x=(A, B)\right.$, params): On input two matrices $A$ and $B$ of respective dimensions $n_{1} \times n_{2}$ and $n_{2} \times n_{3}$, perform:

1. Choose $r_{i} \stackrel{R}{\leftarrow} \mathbb{Z}_{p}^{*}$ for $1 \leq i \leq n_{1}, d_{j}, v_{j}, m_{j}, s_{j} \stackrel{R}{\leftarrow} \mathbb{Z}_{p}^{*}$ for $1 \leq j \leq n_{2}$, and $c_{k}, w_{k}, u_{k} \stackrel{R}{\leftarrow} \mathbb{Z}_{p}^{*}$ for $1 \leq k \leq n_{3}$.

2. Compute $X_{i j}=g_{1}^{r_{i} / m_{j} A_{i j}}$ for $1 \leq i \leq n_{1}$ and $1 \leq j \leq n_{2}$.

3. Compute $Y_{i j}=g_{2}^{c_{j} m_{i} B_{i j}+w_{j} d_{i}}$ for $1 \leq i \leq n_{2}$ and $1 \leq j \leq n_{3}$.

4. Compute $X_{i j}^{\prime}=g_{1}^{r_{i} / v_{j} A_{i j}}$ for $1 \leq i \leq n_{1}$ and $1 \leq j \leq n_{2}$.

5. Compute $Y_{i j}^{\prime}=g_{2}^{c_{j} v_{i} B_{i j}+u_{j} s_{i}}$ for $1 \leq i \leq n_{2}$ and $1 \leq j \leq n_{3}$.

6. Compute $t_{i}=\sum_{k=1}^{n_{2}} A_{i k} d_{k} / m_{k}$ for $1 \leq i \leq n_{1}$, and $\hat{v k_{i j}}=t_{i} r_{i} w_{j}$ for $1 \leq i \leq n_{1}$ and $1 \leq j \leq n_{3}$.

7. Compute $t_{i}^{\prime}=\sum_{k=1}^{n_{2}} A_{i k} s_{k} / v_{k}$ for $1 \leq i \leq n_{1}$, and $v k_{i j}=\hat{v k_{i j}}-t_{i}^{\prime} r_{i} u_{j}$ for $1 \leq i \leq n_{1}$ and $1 \leq j \leq n_{3}$.

8. Compute $s k_{i j}=g_{T}^{v k_{i j}}$ for $1 \leq i \leq n_{1}$ and $1 \leq j \leq n_{3}$, set $s k_{j}=H\left(s k_{1 j}\left\|s k_{2 j}\right\| \ldots \| s k_{n_{1} j}\right)$ for $1 \leq j \leq n_{3}$, and $s k=H\left(s k_{1}\left\|s k_{2}\right\| \ldots \| s k_{n_{3}}\right)$.

9. Set $\mathrm{SK}_{x}=\left(\left\{c_{i}\right\}_{i=1}^{n_{3}},\left\{r_{i}\right\}_{i=1}^{n_{1}},\left\{\hat{v k_{i j}}\right\}_{i=1, j=1}^{n_{1}, n_{3}}, s k\right), \mathrm{EK}_{x}=$ (params), and $\sigma_{x}=$ $\left(X, Y, X^{\prime}, Y^{\prime}\right)$.

Compute $\left(\mathrm{EK}_{x}, \sigma_{x}=\left(X, Y, X^{\prime}, Y^{\prime}\right)\right)$ : Execute the following steps:

1. For $1 \leq j \leq n_{3}$ and $1 \leq i \leq n_{1}$, compute

- $V_{i j}^{(1)}=\prod_{k=1}^{n_{2}} e\left(X_{i k}, Y_{k j}\right)=g_{T}^{\sum_{k=1}^{n_{2}} A_{i k}\left(c_{j} r_{i} B_{k j}+w_{j} d_{k} r_{i} / m_{k}\right)}$

- $V_{i j}^{(2)}=\prod_{k=1}^{n_{2}} e\left(X_{i k}^{\prime}, Y_{k j}^{\prime}\right)=g_{T}^{\sum_{k=1}^{n_{2}} A_{i k}\left(c_{j} r_{i} B_{k j}+u_{j} s_{k} r_{i} / v_{k}\right)}$

- $\Delta_{i j}=V_{i j}^{(1)} / V_{i j}^{(2)}$

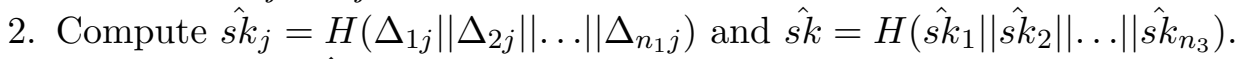

3. Set $\sigma_{y}=\left(V^{(1)}, \hat{s k}\right)$.

$\operatorname{Verify}\left(\mathrm{SK}_{x}=\left(\left\{c_{i}\right\}_{i=1}^{n_{3}},\left\{r_{i}\right\}_{i=1}^{n_{1}},\left\{\hat{v k_{i j}}\right\}_{i=1, j=1}^{n_{1}, n_{3}}, s k\right), \sigma_{y}=\left(V^{(1)}, \hat{s k}\right)\right)$ : Verify whether $\hat{s k}=s k$ and output 1 if the verification was successful and 0 otherwise.

Output $\left(\mathrm{SK}_{x}=\left(\left\{c_{i}\right\}_{i=1}^{n_{3}},\left\{r_{i}\right\}_{i=1}^{n_{1}},\left\{\hat{v k_{i j}}\right\}_{i=1, j=1}^{n_{1}, n_{3}}, s k\right), \sigma_{y}=\left(V^{(1)}, \hat{s k}\right)\right):$ Compute $\quad g_{T}^{C_{i j}}=$ $\left(V_{i j}^{(1)} / g_{T}^{v k_{i j}}\right)^{\left(c_{j} r_{i}\right)^{-1}}$, recover $C_{i j}$ from the result, and output $C$. If the computation fails, output $\perp$.

Figure 5: Description of scheme $\mathrm{VC}_{r}^{p}$ for rational adversaries that achieves data privacy.

passes, the client calls Output and uses $c_{j}$ 's, $r_{i}$ 's, and $v k_{i j}$ 's from its secret key to recover product $C=A \times B$ from $V^{(1)}$. In particular, to obtain each matrix element $C_{i j}$, the client needs to compute the discrete logarithm of $\left(V_{i j}^{(1)} / g_{T}^{\hat{v} k_{i j}}\right)^{\left(c_{j} r_{i}\right)^{-1}}$ to the base $g_{T}$, which in general is hard. However, if we restrict matrix elements to a small range, the discrete logarithm can be computed by trying all values in the range or precomputing all powers of $g_{T}$. The complexity of Output is thus $O\left(n_{1} n_{3}\right)$ cryptographic operations due to the need to recover $C$. 


\subsubsection{Analysis}

To demonstrate correctness, we show that if the computation was performed correctly, the server is able to recover the key $s k$. For each $j=1, \ldots, n_{3}$, we have:

$$
\begin{aligned}
s \hat{k}_{j} & =H\left(\Delta_{1 j}\|\ldots\| \Delta_{n_{1} j}\right)=H\left(V_{1 j}^{(1)} / V_{1 j}^{(2)}\|\ldots\| V_{n_{1} j}^{(1)} / V_{n_{1} j}^{(2)}\right) \\
& =H\left(g_{T}^{\sum_{k=1}^{n_{2}} A_{1 k}\left(w_{j} d_{k} / m_{k}\right) r_{1}-\sum_{k=1}^{n_{2}} A_{1 k}\left(u_{j} s_{k} / v_{k}\right) r_{1}}\|\ldots\| g_{T}^{\sum_{k=1}^{n_{2}} A_{n_{1} k}\left(w_{j} d_{k} / m_{k}\right) r_{n_{1}}-\sum_{k=1}^{n_{2}} A_{n_{1} k}\left(u_{j} s_{k} / v_{k}\right) r_{n_{1}}}\right) \\
& =H\left(\hat{s k}_{1 j} \| \ldots \hat{s k}_{n_{1} j}\right)=s k_{j}
\end{aligned}
$$

Then since $\hat{s k}_{j}=s k_{j}$ for each $j, \hat{s k}=s k$. The data protection property of $\mathrm{VC}_{r}^{p}$ construction can be stated as follows:

Theorem 8 Assuming that the $M-D D H$ and $X D H$ problems are hard and $A$ does not contain zero elements, the verifiable computation scheme $\mathrm{VC}_{r}^{p}$ achieves data secrecy according to Definition $\%$.

Proof We proceed with a series of games using the hybrid argument and analyze the adversary's capabilities in the final game.

Game $\mathbf{G}_{\mathbf{0}}$. Define $\mathbf{G}_{\mathbf{0}}$ to be the same as $\operatorname{Exp}_{\mathcal{A}}^{\text {Priv }}\left(\mathrm{VC}_{r}^{p}, f, \kappa\right)$.

Game $\mathbf{G}_{\mathbf{1}}$. The game is identical to game $\mathbf{G}_{\mathbf{0}}$, except that when generating $Y_{i j}$ s and $Y_{i j}^{\prime}$ s, the delegator will use random values $r_{i j}^{(1)}$ and $r_{i j}^{(2)}$ in $\mathbb{Z}_{p}$ instead of $w_{j} d_{i}$ and $u_{j} s_{i}$, respectively. To be able to verify the result using the original Verify and recover the output using the original Output, the delegator modifies each $v k_{i j}$ to $r_{i} \sum_{k=1}^{n_{2}} A_{i k}\left(r_{k j}^{(1)} / m_{k}-r_{k j}^{(2)} / v_{k}\right)$ and also updates $s k$ correspondingly using the $v k_{i j}$ 's. Moreover, to be able to correctly decrypt the result to recover the output, the delegator also modifies each $\hat{v k}_{i j}$ to $r_{i} \sum_{k=1}^{n_{2}} A_{i k}\left(r_{k j}^{(1)} / m_{k}\right)$. As before, assuming $\left(n_{2}+n_{3}\right)-\mathrm{M}-\mathrm{DDH}$ problem is hard, the difference in the adversary's view between $\mathbf{G}_{\mathbf{1}}$ and $\mathbf{G}_{\mathbf{0}}$ is negligible.

Game $\mathbf{G}_{\mathbf{2}}$. The game is identical to $\mathbf{G}_{\mathbf{1}}$ except that the delegator removes information about $c_{j}$, $B_{i j}$, and $m_{i}\left(v_{i}\right)$ from each $Y_{i j}$ (resp., $\left.Y_{i j}^{\prime}\right)$. Each $Y_{i j}$ is now formed as $g_{1}^{r_{i j}^{(1)}}$ instead of $g_{1}^{c_{j} m_{i} B_{i j}+r_{i j}^{(1)}}$ and each $Y_{i j}^{\prime}$ is now formed as $g_{1}^{r_{i j}^{(2)}}$ instead of $g_{1}^{c_{j} v_{i} B_{i j}+r_{i j}^{(2)}}$. The secret key $\mathrm{SK}_{x}$ will be set to be the same as that of $\mathbf{G}_{\mathbf{1}}$ in order to verify the returned computation, while $\hat{v k_{i j}}$ needs to be modified to $r_{i} \sum_{k=1}^{n_{2}} A_{i k}\left(r_{k j}^{(1)} / m_{k}\right)-c_{j} r_{i} \sum_{k=1}^{n_{2}} A_{i k} B_{k j}$. Because the $r_{i j}^{(1)}$ 's and $r_{i j}^{(2)}$ 's are completely random, the distributions of $Y_{i j}$ 's and $Y_{i j}^{\prime}$ 's in $\mathbf{G}_{\mathbf{1}}$ and their distribution in $\mathbf{G}_{\mathbf{2}}$ are identical and the adversary's view does not change.

Game $\mathbf{G}_{3}$. The game is identical to $\mathbf{G}_{\mathbf{2}}$ except that when generating $X_{i j}$ 's, the delegator will use random values $r_{i j}^{(3)}$ in $\mathbb{Z}_{p}$ instead of $r_{i} / m_{j}$ 's and when generating $X_{i j}^{\prime}$ 's, the delegator will use random values $r_{i j}^{(4)}$ in $\mathbb{Z}_{p}$ instead of $r_{i} / v_{j}$ 's. The delegator then computes $v k_{i j}$ as $\sum_{k=1}^{n_{2}} A_{i k}\left(r_{i k}^{(3)} r_{k j}^{(1)}-r_{i k}^{(4)} r_{k j}^{(2)}\right)$ and $\hat{v k_{i j}}$ as $\sum_{k=1}^{n_{2}} A_{i k} r_{i k}^{(3)} r_{k j}^{(1)}-c_{j} r_{i} \sum_{k=1}^{n_{2}} A_{i k} B_{k j}$. Because some values $\left(r_{i}\right.$ 's) are used in both $X$ and $X^{\prime}$, we treat all $r_{i} / m_{j}$ and $r_{i} / v_{j}$ as an instance of a single M-DDH problem. Then assuming that $\left(n_{1}+2 n_{2}\right)$-M-DDH problem is hard, the difference in the adversary's view between $\mathbf{G}_{2}$ and $\mathbf{G}_{3}$ is negligible.

Game $\mathbf{G}_{\mathbf{4}}$. The game is identical to $\mathbf{G}_{\mathbf{3}}$ except that the delegator removes information about $A_{i j}$ from each $X_{i j}$ and $X_{i j}^{\prime}$, which are now formed as $g_{1}^{r_{i j}^{(3)}}$ and $g_{1}^{r_{i j}^{(4)}}$ instead of $g_{1}^{A_{i j} r_{i j}^{(3)}}$ and $g_{1}^{A_{i j} r_{i j}^{(4)}}$, respectively. The delegator then computes $v k_{i j}$ as $\sum_{k=1}^{n_{2}}\left(r_{i k}^{(3)} r_{k j}^{(1)}-r_{i k}^{(4)} r_{k j}^{(2)}\right)$ and $\hat{v k_{i j}}$ as 
$\sum_{k=1}^{n_{2}} r_{i k}^{(3)} r_{k j}^{(1)}-c_{j} r_{i} \sum_{k=1}^{n_{2}} A_{i k} B_{k j}$. Because the $r_{i j}^{(3)}$ 's and $r_{i j}^{(4)}$ 's are completely random and all $A_{i j}$ 's are non-zero, the distributions of $X_{i j}$ 's and $X_{i j}^{\prime}$ 's in $\mathbf{G}_{\mathbf{3}}$ are identical to their distributions in $\mathbf{G}_{\mathbf{4}}$. Therefore, the adversary's view does not change between $\mathbf{G}_{\mathbf{3}}$ and $\mathbf{G}_{\mathbf{4}}$.

Now observe that we completely removed any information about the input matrices $A$ and $B$ from the adversary's view, which implies that the server's probability in winning in $\mathbf{G}_{\mathbf{4}}$ is exactly $1 / 2$. Combined with the previous analysis of differences in the adversarial success between games $\mathbf{G}_{\mathbf{0}}$ and $\mathbf{G}_{\mathbf{4}}$, we obtain that the adversary's advantage is negligible in winning the experiment $\operatorname{Exp}_{\mathcal{A}}^{\text {Priv }}\left(\mathrm{VC}_{r}^{p}, f, \kappa\right)$.

Theorem 9 If the verification of the returned result was successful, $H$ is a collision-resistant hash function, the $M-D D H$ and XDH assumptions hold, and all elements of $A$ are non-zero, the server that deviates from the correct protocol can only pass verification with a probability negligible in the security parameter.

Proof The only difference in the adversary's view from $\mathrm{VC}_{r}$ is that matrices $(A, B, Z)$ that the adversary receives are replaced with matrices $\left(X^{\prime}, Y^{\prime}\right)$. In either scheme, they are used to form $V^{(1)}$ and consequently construct the verification key $s k$. Next, note that in both schemes the verification key is computed in the same way, but strictly less information about the data is revealed to the adversary in $\mathrm{VC}_{r}^{p}$ (i.e., $X^{\prime}$ and $Y^{\prime}$ do not reveal any information about the key and input matrices under out computational assumptions). Therefore, the adversary does not gain any advantage in passing verification without following the prescribed computation and the security claim holds in $\mathrm{VC}_{r}^{p}$ as well.

Theorem 10 If Assumptions 1 and 2 and assumptions of Theorem 9 hold, $\mathrm{VC}_{r}^{p}$ is secure in presence of rational adversaries according to Definition 6.

As before, the proof will be similar to that of Theorem 3, and we omit the details here. It is worth noting that we can easily modify $\mathrm{VC}_{r}^{p}$ to incorporate key chaining, which allows for efficient location of faulty cells when verification fails, by adding an additional layer of encoding to matrices $Y$ and $Y^{\prime}$.

\section{Support for Public Verifiability}

Recall that public verifiability means that at the time of problem generation the delegator produces a public verification key and after the server executes the computation delegated to it any entity with the knowledge of the public key is able to verify correctness of the outsourced computation. In this section, we incorporate public verifiability into $\mathrm{VC}_{m}$ and $\mathrm{VC}_{r}$ constructions while preserving their original security properties. In particular, the client will now produce a public verification key $\mathrm{PVK}_{x}$ as a public version of $\mathrm{SK}_{x}$ at ProbGen time, which is roughly in the form of $g^{\mathrm{SK}_{x}}$. This key then can be utilized by any auditing entity at Verify time to confirm correctness of outsourced computation upon its completion. We also show how the introduced modifications can be applied to privacy-preserving versions of schemes $\mathrm{VC}_{m}^{p}$ and $\mathrm{VC}_{r}^{p}$ so that all features of public delegatability and verifiability, privacy protection, and computation verification will be supported in a single construction.

\subsection{Support for Public Verifiability in Lazy Setting}

Recall that in $\mathrm{VC}_{r}, S K_{x}$ produced at problem generation time consists of only key $s k$. Therefore, all that is needed to convert the solution into a publicly verifiable scheme is to make a public version 
of the key, $g^{s k}$, publicly available. Thus, our scheme with public verifiability $\mathrm{VC}_{r}^{p v}$ can be described as follows:

1. In ProbGen, the only difference is that in step 7 we set $\mathrm{PVK}_{x}=g^{\text {sk }}$ and the algorithm returns PVK $_{x}$ instead of $\mathrm{SK}_{x}$. Here, $g$ is a generator of a cyclic group $\mathbb{G}$ in which the discrete logarithm problem is hard, and any $\mathbb{G}$ and $g$ will suffice.

2. In Verify, the difference is that the input now includes $\mathrm{PVK}_{x}$ instead of $\mathrm{SK}_{x}$ and the verification consists of checking whether $\mathrm{PVK}_{x}=g^{\hat{s k}}$, where as before $\hat{s k}$ is part of output produced by Compute.

The remaining algorithms, Setup and Compute, are unchanged from $\mathrm{VC}_{r}$.

Theorem 11 Assuming that the $M-D D H$ and $X D H$ problems are hard, $H$ is a collision-resistant hash function, and matrix A contains only non-zero elements, the verifiable computation scheme $\mathrm{VC}_{r}^{p v}$ is secure according to Definition 8.

Proof There is only one difference in the adversary's view between $\mathrm{VC}_{r}$ and $\mathrm{VC}_{r}^{p v}$ in accordance to Definition 8: In $\mathrm{VC}_{r}^{p v}$, the adversary can observe published $\mathrm{PVK}_{x}=g^{s k}$ for all of its queries. However, as the discrete logarithm problem is hard in $\mathbb{G}$, the adversary is unable to extract information about $s k$ and therefore will not gain any non-negligible advantage in winning the security game $\operatorname{Exp}_{\mathcal{A}}^{\text {PubVer }}\left(\mathrm{VC}_{r}^{p v}, f, \kappa\right)$. The proof for this construction proceeds in the same way to the proof of Theorem 2 and the security claim holds.

Because the release of $\mathrm{PVK}_{x}$ does not reveal enough information to compromise security of the scheme, we can apply the same modification to the privacy-preserving version $\mathrm{VC}_{r}^{p}$ of this scheme, without having to make additional adjustments. In the resulting construction, any verifier will be able to assess correctness of the performed computation using $\mathrm{PVK}_{x}$, but will not be able to recover the output without access to additional information stored in $\mathrm{SK}_{x}$. The delegator uses procedure Output to recover the produce matrix $C$.

\subsection{Support for Public Verifiability in Malicious Setting}

Unlike the previous solution, to achieve public verifiability in the malicious adversary setting PVK ${ }_{x}$ to be $g^{v}$ for every $v \in \mathrm{SK}_{x}$ does not work. Doing this would reveal crucial key information, which can be easily exploited by the server to compromise integrity of the computation. In particular, assume that $\mathrm{PVK}_{x}$ now consists $g_{T}^{r_{i} c_{j}}$ for $1 \leq i \leq n_{1}$ and $1 \leq j \leq n_{3}$ and $g_{T}^{\sum_{k=1}^{n_{1}} r_{i} v k_{i}}$, and Verify consists of checking whether $\prod_{i=1}^{n_{1}} \prod_{j=1}^{n_{3}}\left(g_{T}^{r_{i} c_{j}}\right)^{C_{i j}}+g_{T}^{\sum_{k=1}^{n_{1}} r_{i} v k_{i}}=s$ holds (where $s$ is returned by Compute). ${ }^{1}$ To launch an attack, the adversary first correctly computes matrix $C$ and $s$, then randomly chooses one matrix element $C_{i j}$, changes it to $C_{i j}+\delta$, and multiplies $s$ by $g_{T}^{r_{i} c_{j} \delta}$. By doing that, the adversary is able to produce a tuple $(\hat{C}, \hat{s})$ that differs from the correct one $(C, s)$, but nevertheless passes the verification test. Notice that this attack is infeasible in the original $\mathrm{VC}_{m}$ scheme as the adversary has no information about $g_{T}^{r_{i} c_{j}}$ and hence is unable to correctly produce $\hat{s}$. Therefore, to support public verifiability in the malicious setting, we need to introduce further changes to the scheme.

We refer to the new scheme with public verifiability as $\mathrm{VC}_{m}^{p v}$.

\footnotetext{
${ }^{1}$ Note that because $s$ is returned as an element of $\mathbb{G}_{T}$ and each $C_{i j}$ is returned as an element in $\mathbb{Z}_{p}^{*}$, the values $g_{T}^{r_{i} c_{j}}$ for $1 \leq i \leq n_{1}$ and $1 \leq j \leq n_{3}$ and $g_{T}^{\sum_{k=1}^{n_{1}} r_{i} v k_{i}}$ represent the minimum information the verifier needs to possess to carry out the verification procedure.
} 
Setup $\left(1^{\kappa}, f\right)$ : The same as in $\mathrm{VC}_{m}$.

$\operatorname{ProbGen}\left(x=(A, B)\right.$, params): On input two matrices $A$ and $B$ of respective dimensions $n_{1} \times n_{2}$ and $n_{2} \times n_{3}$, perform:

1. Choose $r_{i} \stackrel{R}{\leftarrow} \mathbb{Z}_{p}^{*}$ for $1 \leq i \leq n_{1}, d_{j} \stackrel{R}{\leftarrow} \mathbb{Z}_{p}^{*}$ for $1 \leq j \leq n_{2}$, and $c_{k}, w_{k} \stackrel{R}{\leftarrow} \mathbb{Z}_{p}^{*}$ for $1 \leq k \leq n_{3}$.

2. Compute $t_{i}=\sum_{k=1}^{n_{2}} A_{i k} d_{k}$ for $1 \leq i \leq n_{1}, f=\sum_{j=1}^{n_{3}} w_{j}$, and $v k_{i}=t_{i} f$ for $1 \leq i \leq n_{1}$.

3. Compute $Y_{i j}=g_{2}^{c_{j} B_{i j}+w_{j} d_{i}}$ for $1 \leq i \leq n_{2}$ and $1 \leq j \leq n_{3}$.

4. Set $\mathrm{PVK}_{x}=\left(\left\{g_{1}^{r_{i}}\right\}_{i=1}^{n_{1}},\left\{g_{T}^{r_{i} c_{j}}\right\}_{i=1, j=1}^{n_{1}, n_{3}}, g_{T}^{\sum_{i=1}^{n_{1}} r_{i} v k_{i}}\right), \mathrm{EK}_{x}=$ params, and $\sigma_{x}=(A, B, Y)$.

Compute $\left(\mathrm{EK}_{x}, \sigma_{x}=(A, B, Y)\right)$ : Given $\sigma_{x}$, execute:

1. Compute $C=A \times B$.

2. Compute $s_{i}=\prod_{j=1}^{n_{3}} \prod_{k=1}^{n_{2}} Y_{k j}^{A_{i k}}$ for $1 \leq i \leq n_{1}$.

3. Set $\sigma_{y}=\left(C,\left\{s_{i}\right\}_{i=1}^{n_{1}}\right)$.

$\operatorname{Verify}\left(\mathrm{PVK}_{x}=\left(\left\{g_{1}^{r_{i}}\right\}_{i=1}^{n_{1}},\left\{g_{T}^{r_{i} c_{j}}\right\}_{i=1, j=1}^{n_{1}, n_{3}}, g_{T}^{\sum_{i=1}^{n_{1}} r_{i} v k_{i}}\right), \sigma_{y}=\left(C,\left\{s_{i}\right\}_{i=1}^{n_{1}}\right)\right)$ : Verify $\quad$ whether

$\prod_{i=1}^{n_{1}} \prod_{j=1}^{n_{3}}\left(g_{T}^{r_{i} c_{j}}\right)^{C_{i j}} \times g_{T}^{\sum_{i=1}^{n_{1}} r_{i} v k_{i}}=\prod_{i=1}^{n_{1}} e\left(g_{1}^{r_{i}}, s_{i}\right)$. If the check succeeds, output $C$; otherwise, output $\perp$.

Figure 6: Description of scheme $\mathrm{VC}_{m}^{p v}$ in malicious setting that supports public verifiability.

The idea behind the modification is to prevent the server from merging offset $g_{T}^{r_{i} c_{j} \delta}$ with $s$ by making the values belong to two different groups. That is, we design the scheme in a way that the values of the form $g^{r_{i} c_{j}}$ are available only in $\mathbb{G}_{T}$, while $s$ will have to be produced by the server as an element of $\mathbb{G}_{2}$. Because groups $\mathbb{G}_{2}$ and $\mathbb{G}_{T}$ are used in the bilinear function $e: \mathbb{G}_{1} \times \mathbb{G}_{2} \rightarrow \mathbb{G}_{T}$, there does not exist an efficiently computable conversion from an element $g_{T}^{x}$ in $\mathbb{G}_{T}$ to the corresponding element $g_{2}^{x}$ in $\mathbb{G}_{2}$. Then in the construction the delegator produces PVK $x$ that consists of $g_{1}^{r_{i}}, g_{T}^{r_{i} c_{j}}$ and $g_{T}^{\sum_{i=1}^{n_{1}} r_{i} v k_{i}}$ and provides $\sigma_{x}$ that consists of only three input matrices $A, B$, and $Y$ (rather than four in $\mathrm{VC}_{m}$ ) to the server. The server computes each $s_{i}$ to be an element of $\mathbb{G}_{2}$ by performing a modulo exponentiation using $A$ and $Y$ (rather than the pairing operation in $\mathrm{VC}_{m}$ ) and returns their values to the client. Because verification of the result now consists of checking whether the product of all $\left(g_{T}^{r_{i} c_{j}}\right)^{C_{i j}}$ 's and $g_{T}^{\sum_{i=1}^{n_{1}} r_{i} v k_{i}}$ matches the product of $e\left(g_{1}^{r_{i}}, s_{i}\right)$ 's, it is no longer feasible for the adversary to succeed in the above attack. A detailed description of the new scheme $\mathrm{VC}_{m}^{p v}$ is given in Figure 6.

The complexity of ProbGen is dominated by computing public verification key $\mathrm{PVK}_{x}$ and matrix $Y$, and is $O\left(n_{1} n_{2}+n_{1} n_{3}+n_{2} n_{3}\right)$. Compute involves matrix multiplication and has complexity $O\left(n_{1} n_{2} n_{3}\right)$. Verify consists of ensuring the validity of $C$ by checking its elements against $s_{i}$ that Compute produces and involves $n_{1} n_{3}$ modulo exponentiations and $n_{1}$ pairing operations.

Theorem 12 Assuming that the $M-D D H, X D H$, and some other problems are hard, the verifiable computation scheme with public verifiability $\mathrm{VC}_{m}^{p v}$ is secure according to Definition 8.

Proof Similar to previous proofs, we proceed with a series of games using the hybrid argument and analyze the adversary's capabilities in the final game.

Game $\mathbf{G}_{\mathbf{0}}$. Define $\mathbf{G}_{\mathbf{0}}$ to be the same as $\operatorname{Exp}_{\mathcal{A}}^{\text {PubVer }}\left(\mathrm{VC}_{m}^{p v}, f, \kappa\right)$. 
Game $\mathbf{G}_{\mathbf{1}}$. The game is identical to game $\mathbf{G}_{\mathbf{0}}$, except that when generating $Y_{i j}$ and $v k$ in $g_{T}^{v k}$, the delegator will use a random value $r_{i j}^{(1)}$ from $\mathbb{Z}_{p}$ instead of $w_{j} d_{i}$. Assuming that $\left(n_{2}+n_{3}\right)-\mathrm{M}-\mathrm{DDH}$ problem is hard, the difference in the adversary's view between $\mathbf{G}_{\mathbf{1}}$ and $\mathbf{G}_{\mathbf{0}}$ is negligible.

Game $\mathbf{G}_{\mathbf{2}}$. We next remove information about $c_{j}$ 's from the elements of $Y$. That is, we replace each $Y_{i j}=g_{2}^{r_{i j}^{(1)}+c_{j} B_{i j}}$ with $g_{2}^{r_{i j}^{(1)}}$. Because each $r_{i j}^{(1)}$ is random, the distribution of $Y_{i j}$ 's will not be affected. The $r_{i j}^{(1)}$ 's, however, are also used in $v k$ and thus we also update $g_{T}^{v k}$ to be consistent with other information. That is, $g_{T}^{v k}$ from $\mathbf{G}_{\mathbf{1}}$ is multiplied by $g_{T}^{-\sum_{i=1}^{n_{1}} \sum_{j=1}^{n_{3}} \sum_{k=1}^{n_{2}} r_{i} c_{j} A_{i k} B_{k j}}$ to form $g_{T}^{v k}=g_{T}^{\sum_{i=1}^{n_{1}} \sum_{j=1}^{n_{3}} \sum_{k=1}^{n_{2}} r_{i} A_{i k}\left(r_{k j}^{(1)}-c_{j} B_{k j}\right)}$ in $\mathbf{G}_{\mathbf{2}}$.

Game $\mathbf{G}_{\mathbf{3}}$. In this game, we apply another instance of M-DDH problem. This time, we replace all instances of $r_{i} c_{j}$ (in $g_{T}^{r_{i} c_{j}}$ ) with random values from $\mathbb{Z}_{p}$, which we denote by $r_{i j}^{(2)}$.

That is, now each $g_{T}^{r_{i} c_{j}}$ in $\mathrm{PVK}_{x}$ is replaced with $g_{T}^{r_{i j}^{(2)}}$ and $g_{T}^{v k}$ is replaced with $g_{T}^{\sum_{i=1}^{n_{1}} \sum_{j=1}^{n_{3}} \sum_{k=1}^{n_{2}} A_{i k}\left(r_{i} r_{k j}^{(1)}-r_{i j}^{(2)} B_{k j}\right)}$. Assuming that $\left(n_{3}+n_{1}\right)$-M-DDH problem is hard, the difference in the adversary's view between $\mathbf{G}_{\mathbf{2}}$ and $\mathbf{G}_{\mathbf{1}}$ is negligible. Note that the M-DDH assumption holds when $g_{T}^{c_{i}}$ and $g_{T}^{r_{i}}$ are available to the adversary. In our case, the adversary has access to $g_{1}^{r_{i}}$ and therefore $g_{T}^{r_{i}}$, but no information about $g_{2}^{c_{j}}$ is available, which means that we can safely apply the M-DDH assumption.

Now, suppose that in game $\mathbf{G}_{\mathbf{3}}$ the server is able to return output $\left(\hat{C},\left\{\hat{s}_{i}\right\}_{i=1}^{n_{1}}\right)$ that differs from the expected $\left(C,\left\{s_{i}\right\}_{i=1}^{n_{1}}\right)$, but which nevertheless passes verification. According to the security definition, the adversary wins if $\hat{C} \neq C$, but verification is successful. This means that the returned values must satisfy $\prod_{i=1}^{n_{1}} \prod_{j=1}^{n_{3}}\left(g_{T}^{r_{i j}^{(2)}}\right)^{C_{i j}-\hat{C}_{i j}}=\prod_{i=1}^{n_{1}} e\left(g_{1}^{r_{i}}, s_{i}-\hat{s}_{i}\right)$, where $C_{i j} \neq \hat{C}_{i j}$ for at least one value of $i$ and $j$.

Now note that the server has no information regarding $r_{i j}^{(2)}$, s beyond the released $g_{T}^{r_{i j}^{(2)}}$, s and thus he has a negligible probability of making $\sum_{i=1}^{n_{1}} \sum_{j=1}^{n_{3}} r_{i j}^{(2)}\left(C_{i j}-\hat{C}_{i j}\right)$ equal to any given value beyond his control. Furthermore, the only information available to the server in $\mathbb{G}_{2}$ are random elements of the group $g_{2}^{r_{i j}^{(1)}}$ (which in particular means that the $\hat{s}_{i}$ 's cannot depend on $r_{i j}^{(2)}$, s as the server is unable to use $g_{T}^{r_{i j}^{(2)}}$ 's from $\mathbb{G}_{T}$ to form $\hat{s}_{i}$ 's in $\mathbb{G}_{2}$ ).

This means that in this game the server must guess a function of random elements from $\mathbb{Z}_{p}$ when forming $\hat{C} \neq C$, the probability of which is negligible in the security parameter and thus the probability of winning the game.

Combined with the previous analysis of the differences in the adversarial success between games $\mathbf{G}_{\mathbf{0}}$ and $\mathbf{G}_{\mathbf{3}}$, we obtain that $\mathcal{A}$ 's advantage is negligible in winning the experiment $\mathbf{E x p}_{\mathcal{A}}^{\text {PubVer }}\left(\mathbf{V} C_{m}^{p v}, f, \kappa\right)$, as desired.

While the above result already states security of the scheme, we can prove an even stronger property which guarantees that all information returned by the server, i.e., both matrix $C$ and vector $s$, is correct. This is opposed to only guaranteeing that the output of the computation $C$ is correct. To show the strengthen result, we rely on a new cryptographic assumption that we call $k$-Computational Linear Aggregation (CLA) assumption and which we prove secure in the generic group model. We obtain the following:

Theorem 13 Assuming that the $M-D D H, X D H$, and CLA problems are hard, the verifiable computation scheme with public verifiability $\mathrm{VC}_{m}^{p v}$ is secure according to Definition 8 and additionally guarantees that all information returned by malicious server in $\sigma_{y}$ is correct. 
The details of the $k$-CLA assumption, its proof of security in the generic model, and the proof of Theorem 13 can be found in Appendix B.

\subsection{Support for Public Verifiability and Data Privacy in Malicious Setting}

Recall that it was easy to add data privacy to scheme $\mathrm{VC}_{m}$ because the computation consisted of computing products $A \times B$ and $X \times Y$. These products can be computed independently and therefore we could encode matrices $A$ and $B$ using any homomorphic encryption scheme that supports one multiplication and many additions on encrypted data. In $\mathrm{VC}_{m}^{p v}$, on the other hand, matrix $X$ is not used, but instead both products utilize $A$. Thus, if we encrypt input matrices $A$ and $B$ in order to protect the data from the computational server and the auditor, the client will need to incorporate randomness used during forming ciphertexts into public verification key $\mathrm{PVK}_{x}$, which makes the use of encryption more challenging. More importantly, to produce $s_{i}$ 's to be in $\mathbb{G}_{2}$, we need the elements of $A$ to take values from $\mathbb{Z}_{p}$ because $Y_{i j}$ (which are also used in the computation of $s_{i}$ 's) are already elements of $\mathbb{G}_{2}$. To meet the above requirements, instead of using encryption, we additively split each element of matrix $A$ into two shares to obtain matrices $A^{0}$ and $A^{1}$ that will be distributed to independent and non-colluding servers. The same is applied to matrix $B$. The total of four servers are used. Each server then performs the same computation as in $\mathrm{VC}_{m}^{p v}$ on its shares of matrices $A$ and $B$ and each result is verified independently.

When the client receives the results from all servers, it can reconstruct the output from its shares. More specifically, the solution proceeds as follows:

1. The Setup procedure is the same as in $\mathrm{VC}_{m}^{p v}$.

2. At ProbGen time, the client performs the following steps:

(a) Generate a $n_{1} \times n_{2}$ matrix $R$ with its elements randomly drawn from $\{0,1\}^{\ell+\rho}$, where $\ell$ is the bitlength of the elements of $A$ and $B$ and $\rho$ is a statistical security parameter. Use matrix $R$ to split matrix $A$ into $A^{0}$ and $A^{1}$ with their elements computed as $A_{i j}^{0}=R_{i j}$ and $A_{i j}^{1}=A_{i j}-R_{i j}$, respectively. Similarly, generate a $n_{2} \times n_{3}$ matrix $R^{\prime}$ with its elements drawn at random from $\{0,1\}^{\ell+\rho}$ and split matrix $B$ into $B^{0}$ and $B^{1}$ as $B_{i j}^{0}=R_{i j}^{\prime}$ and $B_{i j}^{1}=B_{i j}-R_{i j}^{\prime}$.

(b) Set $\mathrm{EK}_{x}$ computed as in $\mathrm{VC}_{m}^{p v}$, and $\sigma_{x}=\left(A^{0}, B^{0}, Y^{0}\right)$ for the first server, $\sigma_{x}=\left(A^{1}, B^{0}, Y^{0}\right)$ for the second server, $\sigma_{x}=\left(A^{0}, B^{1}, Y^{1}\right)$ for the third server, and $\sigma_{x}=\left(A^{1}, B^{1}, Y^{1}\right)$ for the fourth server, where $Y^{i}$ denotes the matrix $Y$ computed according to the elements of $B^{i}$. $\mathrm{PVK}_{x}$ is also computed in the same way as in $\mathrm{VC}_{m}^{p v}$ with the exception that $g_{T}^{v k}$ is computed according to $A^{0}$ in $\mathrm{PVK}_{x}$ for the first and third servers and according to $A^{1}$ for the second and fourth servers.

3. For Compute, each server performs the same computation as in $\mathrm{VC}_{m}^{p v}$ on its respective inputs.

4. During Verify, an auditor uses $\sigma_{y}$ returned by an individual server and the corresponding $\mathrm{PVK}_{x}$ to verify the returned result in the same way as in $\mathrm{VC}_{m}^{p v}$.

5. During Output, the client receives $\sigma_{y}$ from each of the four servers. Let $C^{i}$ denote the product matrix returned by server $i$. The client then recovers $C$ by computing $C^{1}+C^{2}+C^{3}+C^{4}=$ $A \times B$.

Theorem 14 Assuming the $M-D D H$ and XDH problems are hard and independent and non-colluding servers and auditors are used, the above verifiable computation scheme with public verifiability and data secrecy $\mathrm{VC}_{m}^{p v, p}$ is a secure according to Definitions 7 and 8. 
The proof of this theorem is straightforward. That is, $\mathrm{VC}_{m}^{p v}$ is used without changes, which means that $\mathrm{VC}_{m}^{p v, p}$ is also a verifiable computation scheme with public verifiability. Data privacy follows from the fact that a server or an auditor only obtain access to statistically protected data and the unprotected data is never used in the computation. Thus, the probability of the adversary learning any information about the data is negligible in the statistical security parameter $\rho$.

\section{Conclusions}

This work presents schemes for verifiable outsourcing of matrix multiplications, where it is assumed that the server to which the computation is being outsourced can either arbitrarily deviate from the prescribed computation or is rational, or lazy, in the sense that it will perform only the minimum amount of work to pass the verification but will not maliciously corrupt the returned result. The complexity and features of our schemes favorably compare to the state of the art, with the solution in the lazy setting having lower verification cost of only a single comparison. Our basic constructions achieve public delegatability and can be extended with features of data protection, public verifiability and chaining (supporting all or a subset of the features).

\section{References}

[1] S. Agrawal and D. Boneh. Homomorphic MACs: MAC-based integrity for network coding. In ACNS, pages 292-305, 2009.

[2] B. Applebaum, Y. Ishai, and E. Kushilevitz. From secrecy to soundness: Efficient verification via secure computation. In ICALP, pages 152-163, 2010.

[3] M. Atallah and M. Blanton, editors. Algorithms and Theory of Computation Handbook. Volume I: General Concepts and Techniques, chapter 17. CRC Press, 2009.

[4] M. Atallah and K. Frikken. Securely outsourcing linear algebra computations. In ASIACCS, pages $48-59,2010$.

[5] M. Atallah, K. Frikken, and S. Wang. Private outsourcing of matrix multiplication over closed semi-rings. In SECRYPT, pages 136-144, 2012.

[6] P. D. Azar and S. Micali. Rational proofs. In Proceedings of the 44th Annual ACM Symposium on Theory of Computing, STOC '12, pages 1017-1028, 2012.

[7] P. D. Azar and S. Micali. Super efficient rational proofs. In Proceedings of the 14th ACM Conference on Electronic Commerce, EC '13, pages 29-30, 2013.

[8] L. Babai. Trading group theory for randomness. In STOC, pages 421-429, 1985.

[9] M. Backes, D. Fiore, and R. M. Reischuk. Verifiable delegation of computation on outsourced data. In $C C S$, pages 863-874, 2013.

[10] L. Ballard, M. Green, B. Medeiros, and F. Monrose. Correlation-resistant storage via keywordsearchable encryption. IACR Cryptology ePrint Archive Report 2005/417, 2005.

[11] N. Bitansky, R. Canetti, A. Chiesa, and E. Tromer. From extractable collision resistance to succinct non-interactive arguments of knowledge, and back again. In ITCS, pages 326-349, 2012 . 
[12] N. Bitansky, R. Canetti, A. Chiesa, and E. Tromer. Recursive composition and bootstrapping for snarks and proof-carrying data. In Proceedings of the 45th Annual ACM Symposium on Theory of Computing, STOC '13, pages 111-120, New York, NY, USA, 2013. ACM.

[13] D. Boneh and D. M. Freeman. Homomorphic signatures for polynomial functions. In Proceedings of the 30th Annual International Conference on Theory and Applications of Cryptographic Techniques, EUROCRYPT'11, pages 149-168, Berlin, Heidelberg, 2011. Springer-Verlag.

[14] D. Boneh and D. M. Freeman. Linearly homomorphic signatures over binary fields and new tools for lattice-based signatures. In Proceedings of the 14th International Conference on Practice and Theory in Public-Key Cryptography, PKC'11, pages 1-16, Berlin, Heidelberg, 2011. Springer-Verlag.

[15] D. Boneh, E. Goh, and K. Nissim. Evaluating 2-dnf formulas on ciphertexts. In Proceedings of the Second International Conference on Theory of Cryptography, TCC'05, pages 325-341, 2005 .

[16] E. Bresson, O. Chevassut, and D. Pointcheval. Dynamic group diffie-hellman key exchange under standard assumptions. In Proceedings of the 21st International Conference on the Theory and Applications of Cryptographic Techniques, EUROCRYPT '02, pages 321-336, 2002.

[17] D. Catalano and D. Fiore. Practical homomorphic macs for arithmetic circuits. In Proceedings of the 32nd Annual International Conference on Theory and Applications of Cryptographic Techniques, EUROCRYPT'13, pages 336-352, Berlin, Heidelberg, 2013. Springer-Verlag.

[18] D. Catalano, D. Fiore, R. Gennaro, and K. Vamvourellis. Algebraic (trapdoor) one-way functions and their applications. In $T C C$, pages 680-699, 2013.

[19] D. Catalano, D. Fiore, and B. Warinschi. Efficient network coding signatures in the standard model. In Proceedings of the 15th International Conference on Practice and Theory in PublicKey Cryptography, PKC'12, pages 680-696, Berlin, Heidelberg, 2012. Springer-Verlag.

[20] CertiVox. Benchmarks and Subs performance for MIRACL library. https://certivox.org/display/EXT/Benchmarks+and+Subs.

[21] K. Chung, Y. T. Kalai, F. Liu, and R. Raz. Memory delegation. In CRYPTO, pages 151-165, 2011.

[22] K.-M. Chung, Y. Kalai, and S. Vadhan. Improved delegation of computation using fully homomorphic encryption. In CRYPTO, pages 483-501, 2010.

[23] Y. Dodis, S. Halevi, and T. Rabin. A cryptographic solution to a game theoretic problem. In Proceedings of the 20th Annual International Cryptology Conference on Advances in Cryptology, CRYPTO '00, pages 112-130, 2000.

[24] D. Fiore and R. Gennaro. Publicly verifiable delegation of large polynomials and matrix computations, with applications. In CCS, pages 501-512, 2012.

[25] J. Garay, J. Katz, U. Maurer, B. Tackmann, and V. Zikas. Rational protocol design: Cryptography against incentive-driven adversaries. In Proceedings of the 54th Annual IEEE Symposium on Foundations of Computer Science, FOCS 2013, pages 648-657, 2013. 
[26] R. Gennaro, C. Gentry, and B. Parno. Non-interactive verifiable computing: outsourcing computation to untrusted workers. In CRYPTO, pages 465-482, 2010.

[27] R. Gennaro and D. Wichs. Fully homomorphic message authenticators. Cryptology ePrint Archive, Report 2012/290, 2012.

[28] C. Gentry. Fully homomorphic encryption using ideal lattices. In STOC, pages 169-178, 2009.

[29] C. Gentry and D. Wichs. Separating succinct non-interactive arguments from all falsifiable assumptions. In STOC, pages 99-108, 2011.

[30] S. Goldwasser, Y. T. Kalai, and G. N. Rothblum. Delegating computation: Interactive proofs for muggles. In STOC, pages 113-122, 2008.

[31] S. Goldwasser, S. Micali., and C. Rackoff. The knowledge complexity of interactive proof systems. SIAM Journal on Computing, 18(1):186-208, Feb 1989.

[32] S. Guo, S. Hubáček, A. Rosen, and M. Vald. Rational arguments: Single round delegation with sublinear verification. In Proceedings of the 5th Conference on Innovations in Theoretical Computer Science, ITCS '14, pages 523-540, 2014.

[33] J. Halpern and V. Teague. Rational secret sharing and multiparty computation: Extended abstract. In Proceedings of the 36th Annual ACM Symposium on Theory of Computing, STOC '04, pages 623-632, 2004.

[34] R. Johnson, D. Molnar, D. X. Song, and D. Wagner. Homomorphic signature schemes. In $C T$-RSA, pages 244-262, 2002.

[35] J. Kilian. A note on efficient zero-knowledge proofs and arguments (extended abstract). In STOC, pages 723-732, 1992.

[36] J. Kilian. Improved efficient arguments. In CRYPTO, pages 311-324, 1995.

[37] J. Landerman, V. Pan, and X.-H. Sha. On practical algorithms for accelerated matrix mutiplication. Linear Algebra and Its Applications, 162-164:557-588.

[38] S. Micali. CS proofs. In FOCS, pages 436-453, 1994.

[39] P. Mohassel. Efficient and secure delegation of linear algebra. IACR Cryptology ePrint Archive Report 2011/605, 2011.

[40] B. Parno, J. Howell, C. Gentry, and M. Raykova. Pinocchio: Nearly practical verifiable computation. In IEEE Symposium on Security and Privacy, pages 238-252, 2013.

[41] B. Parno, M. Raykova, and V. Vaikuntanathan. How to delegate and verify in public: Verifiable computation from attribute-based encryption. In TCC, pages 422-439, 2012.

[42] S. Setty, , N. Panpalia, B. Braun, A. J. Blumberg, and M. Walfish. Taking proof-based verified computation a few steps closer to practicality. In Proceedings of the 21st USENIX Conference on Security Symposium, Security'12, pages 253-268, Berkeley, CA, USA, 2012. USENIX Association.

[43] S. Setty, R. McPherson, A. Blumberg, and M. Walfish. Making argument systems for outsourced computation practical (sometimes). In Proceedings of the Network and Distributed Systems Security Symposium, NDSS'12, 2012. 
[44] V. Shoup. Lower bounds for discrete logarithms and related problems. In Proceedings of the 16th Annual International Conference on Theory and Application of Cryptographic Techniques, EUROCRYPT'97, pages 256-266, Berlin, Heidelberg, 1997. Springer-Verlag.

[45] M. D. Van, A. Juels, A. Oprea, R. L. Rivest, E. Stefanov, and N. Triandopoulos. Hourglass schemes: How to prove that cloud files are encrypted. In $C C S$, pages 265-280, 2012.

[46] V. Vu, S. Setty, A. Blumberg, and M. Walfish. A hybrid architecture for interactive verifiable computation. In Proceedings of the 2013 IEEE Symposium on Security and Privacy, SP'13, Berkeley, CA, USA, 2013. IEEE Computer Society.

[47] L. Zhang and R. Safavi-Naini. Private outsourcing of polynomial evaluation and matrix multiplication using multilinear maps. In Cryptology and Network Security, pages 329-348. Springer International Publishing, 2013.

\section{A Additional Definitions}

We present the security experiment for a verifiable scheme with public verifiability:

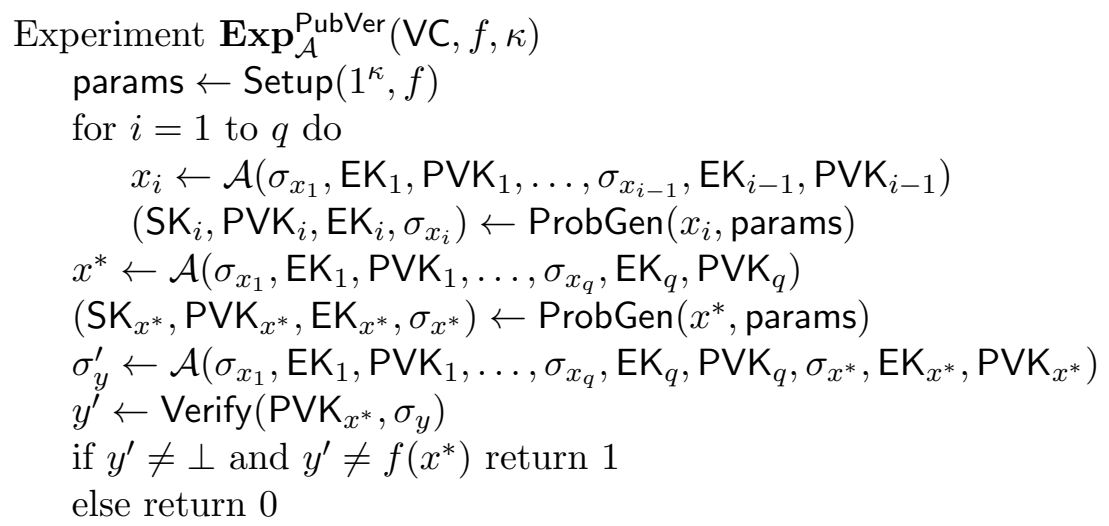

\section{B Stronger Security of $\mathrm{VC}_{m}^{p v}$ and $k$-CLA Assumption}

In this section, we introduce a new assumption, called $k$-Computational Linear Aggregation $(k$ CLA) assumption, show its security in the generic group model, and then show that utilizing it will allow us to prove a stronger security property of the verifiable computation scheme with public verifiability in presence of malicious adversaries $\mathrm{VC}_{m}^{p v}$ as described in Section 7.2.

The $k$-CLA assumption is defined as follows:

Definition 9 (k-CLA assumption) $\operatorname{Let}\left(p, \mathbb{G}_{1}, \mathbb{G}_{2}, \mathbb{G}_{T}, g_{1}, g_{2}, e\right) \leftarrow \operatorname{Set}\left(1^{\kappa}\right)$. Given $R=\left(g_{1}^{r_{1}}, g_{1}^{r_{2}}\right.$, $\left.\ldots, g_{1}^{r_{k}}\right)$ with random $r_{i} \in \mathbb{Z}_{p}^{*}$, the adversary $\mathcal{A}$ needs to produce $T=\left(g_{2}^{t_{1}}, g_{2}^{t_{2}}, \ldots, g_{2}^{t_{k}}\right)$ with $t_{i} \in \mathbb{Z}_{p}^{*}$ that satisfy the equation $\sum_{i=1}^{k} r_{i} t_{i} \equiv 0(\bmod p)$. A's advantage in solving the $k$-CLA problem is defined as

$$
\boldsymbol{A} \boldsymbol{d} \boldsymbol{v}_{\mathcal{A}}^{k-\mathrm{CLA}}(\kappa)=\operatorname{Pr}\left[\mathcal{A}\left(p, g_{1}, g_{2}, k, e, D\right)=1\right] .
$$

We say that the $k$-CLA assumption holds if for every PPT algorithm $\mathcal{A}, \boldsymbol{A d v}_{\mathcal{A}}^{k-C L A}(\kappa)$ is negligible in $\kappa$.

Lemma 2 In the generic group model, the probability for an adversary who issues $q$ operations to solve the $k$-CLA problem is at most $\left(k^{2}+4 q^{2}+2 k q+k+4\right) / 2 p$. 
Proof Given public information $p, \mathbb{G}_{1}, \mathbb{G}_{2}, \mathbb{G}_{T}, g_{1}, g_{2}, e$, and $k$, we keep track of three lists, $L_{1}=\left\{\left(x_{i}, \sigma_{1}\left(x_{i}\right)\right)\right\}, L_{2}=\left\{\left(y_{i}, \sigma_{2}\left(y_{i}\right)\right)\right\}$, and $L_{3}=\left\{\left(z_{i}, \sigma_{3}\left(z_{i}\right)\right)\right\}$ with each of them maintaining the queries performed on cyclic groups $\mathbb{G}_{1}, \mathbb{G}_{2}$, and $\mathbb{G}_{T}$, respectively. Initially, $L_{1}$ contains $k+1$ encodings corresponding to the group generator $g_{1}$ and inputs $g_{1}^{r_{i}}$ for $1 \leq i \leq k, L_{2}$ contains a single encoding corresponding to the group generator $g_{2}$, and $L_{3}$ is empty. The three lists will be updated according to the procedures listed below:

- Group Action in $\mathbb{G}_{1}$ : The generic algorithm specifies two values $x_{i}$ and $x_{j}$ of the current encoding list $L_{1}$ and a sign bit, and the oracle computes $x^{\prime}=x_{i}+x_{j}$ or $x^{\prime}=x_{i}-x_{j}$ according to the sign bit. If $\left(x^{\prime}, \sigma_{1}\left(x^{\prime}\right)\right) \in L_{1}$, the oracle will return $\sigma_{1}\left(x^{\prime}\right)$ to the algorithm; otherwise, the oracle will choose a new value for $\sigma_{1}\left(x^{\prime}\right)$, append it to $L_{1}$, and return it to the algorithm.

- Group Action in $\mathbb{G}_{2}$ : This procedure is the same as for $\mathbb{G}_{1}$, except that the queried inputs are encoded using function $\sigma_{2}$ and list $L_{2}$ is used instead of $L_{1}$.

- Group Action in $\mathbb{G}_{T}$ : This procedure is the same as for $\mathbb{G}_{1}$, except that the queried inputs are encoded using function $\sigma_{3}$ and list $L_{3}$ is used instead of $L_{1}$.

- Bilinear Map: The generic algorithm specifies two values $x_{i}$ and $y_{j}$ from lists $L_{1}$ and $L_{2}$, respectively, and the oracle will compute $z_{i j}=x_{i} \cdot y_{j}$. If $\left(z_{i j}, \sigma_{3}\left(z_{i j}\right)\right) \in L_{3}$, the oracle will return $\sigma_{3}\left(z_{i j}\right)$ to the algorithm; otherwise, the oracle will choose a new value for $\sigma_{3}\left(z_{i j}\right)$, append it to $L_{3}$, and return it to the algorithm.

After performing $q$ operations based on the above rules, the algorithm outputs a list $T=\left(\sigma_{2}\left(y_{1}\right)\right.$, $\left.\sigma_{2}\left(y_{2}\right), \ldots, \sigma_{2}\left(y_{k}\right)\right)$. Because the encodings for the elements in each cyclic group are represented as random polynomials, their degree will be at most 1 in $L_{1}$ and $L_{2}$ and at most 2 in $L_{3}$. Furthermore, it was shown in [44] that (i) a non-zero polynomial with degree $d$ in the group of order $p$ will be evaluated to 0 with probability at most $d / p$, and (ii) two unequal polynomials of degree $d$ instantiated with random values for their variables will be evaluated to the same value with probability at most $d / p$. Next, we analyze the probability of simulation failure that occurs when the oracle outputs two different encodings for the same element in the group, namely two unequal polynomials in $L_{1}, L_{2}$, and $L_{3}$ evaluate to the same value when the variables are assigned random values from $\mathbb{Z}_{p}$. There may occur four inconsistencies:

1. The first inconsistency may occur in the encodings of elements in $\mathbb{G}_{1}$, i.e., when two distinct polynomials originated from the encoding $\sigma_{1}$ evaluate to the same output on inputs chosen uniformly at random. Based on [44], the probability that this happens is at most $1 / p$. Then if $\tau_{1}$ queries were issued in $\mathbb{G}_{1}$, there will be $\left(k+\tau_{1}+1\right)$ pairs in $L_{1}$. The probability that this inconsistency occurs for any element in $L_{1}$ is $\left(\begin{array}{c}\tau_{1}+k+1 \\ 2\end{array}\right)=\frac{\left(k+\tau_{1}+1\right)\left(k+\tau_{1}\right)}{2 p} \leq \frac{(k+q+1)(k+q)}{2 p}$.

2. The second inconsistency may occur in the encodings of elements in $\mathbb{G}_{2}$. Similar to the previous case, the probability of this occurring for any two elements in $L_{2}$ is $1 / p$. If $\tau_{2}$ queries were issued in $\mathbb{G}_{2}$, there will be $\left(\tau_{2}+1\right)$ pairs in $L_{2}$, and the probability of this inconsistency occurring for any element in $L_{2}$ is $\left(\begin{array}{c}\tau_{2}+1 \\ 2\end{array}\right)=\frac{\left(\tau_{2}+1\right) \tau_{2}}{2 p} \leq \frac{q(q+1)}{2 p}$.

3. The third inconsistency may occur in the encodings of elements in $\mathbb{G}_{T}$. Unlike the previous two cases, the degree of polynomials for the encoding $\sigma_{3}$ has degree at most 2 , and therefore the probability that two specific polynomials result in this inconsistency is $\leq 2 / p$. When $\tau_{3}$ queries were issued in $\mathbb{G}_{T}$, there will be $\left(\tau_{3}\right)$ pairs in $L_{3}$, and the probability of this inconsistency occurring for any element in $L_{3}$ is $\left(\begin{array}{c}\tau_{3} \\ 2\end{array}\right)=\frac{\tau_{3}\left(\tau_{3}-1\right)}{p} \leq \frac{q(q-1)}{p}$. 
4. Finally, we would like to show that it is unlikely that polynomials originated from encodings $\sigma_{1}$ and $\sigma_{2}$ satisfy the equation $\sum_{i=1}^{k} \sigma_{1}\left(x_{i}\right) \sigma_{2}\left(y_{i}\right)=0(\bmod p)$, which implies that the answers returned by the adversary do not satisfy the $k$-CLA problem. In particular, the polynomial $\sum_{i=1}^{k} \sigma_{1}\left(x_{i}\right) \sigma_{2}\left(y_{i}\right)$ has degree at most 2 and will not be a zero-polynomial. Therefore, when the variables are instantiated with random values, the probability for the polynomial to be evaluated to zero is $\leq 2 / p$. This means that when the adversary returns a single assignment to solve the $k$-CLA problem, the probability for the occurrence of any inconsistency in the assignment will be $\leq 2 / p$.

We obtain that, based on the union bound, the probability for any of the above inconsistencies to occur is $\leq\left(k^{2}+4 q^{2}+2 k q+k+4\right) / 2 p \square$.

Proof of Theorem 13 Consider the setup used in the proof of Theorem 12. It was already shown that if the server modifies matrix product $C$, verification will be successful only with a negligible probability. To prove the stronger statement of this theorem, we now need to show that if the server returns correct matrix $C$, but incorrect values for $s_{i}$ 's, it is also unable to pass the verification with more than a negligible probability.

Suppose that the server returns correct matrix $C$. In order for the returned values to deviate from the correct values, there should exist at least a single $\hat{s}_{i}$ for some $1 \leq i \leq n_{1}$ that is not equal to $s_{i}$. Let the number of $\hat{s}_{i}$ 's that are not not equal to the corresponding $s_{i}$ 's to be $\mathrm{k}$ for some $1 \leq k \leq n_{1}$. Because the verification procedure checks for $g_{T}^{v k} \prod_{i=1}^{n_{1}} \prod_{j=1}^{n_{3}}\left(g_{T}^{r_{i} c_{j}}\right)^{C_{i j}}=\prod_{i=1}^{n_{1}} e\left(g_{1}^{r_{i}}, s_{i}\right)$, we obtain that in order to pass verification, it must hold that $\prod_{i=1}^{k} e\left(g_{1}^{r_{i}}, \Delta s_{i}\right)=g_{T}^{0}$, where $\Delta s_{i}$ is the difference between $s_{i}$ and $\hat{s}_{i}$. Recall that each $s_{i}$ is in the form $g_{2}^{u_{i}}$ for some $u_{i}$ and thus $\Delta s_{i}$ can be written as $g_{2}^{u_{i}-u_{i}^{\prime}}=g_{2}^{t_{i}}$. Now, given $g_{1}^{r_{i}}$ for $i=1, \ldots, n_{1}$, the server needs to compute $g^{t_{i}}$ for $1 \leq i \leq k$ such that each $t_{i}$ is non-zero and the equation $\prod_{i=1}^{k} e\left(g_{1}^{r_{i}}, g_{2}^{t_{i}}\right)=g_{T}^{0}$ holds. Note that this is exactly what the $k$-CLA problem is. Thus, assuming that $n_{1}$-CLA assumption is hard, we obtain that the server will not be able to corrupt any information in the output without being noticed with more than a negligible probability. 